\title{
cAMP Response Element Binding Protein Is Required for Differentiation of Respiratory Epithelium during Murine Development
}

\author{
A. Daniel Bird ${ }^{1 *}$, Sharon J. Flecknoe ${ }^{2}$, Kheng H. Tan ${ }^{1}$, P. Fredrik Olsson ${ }^{1}$, Nisha Antony ${ }^{1}$, Theo \\ Mantamadiotis $^{3}$, Stuart B. Hooper ${ }^{2}$, Timothy J. Cole'
}

1 Department of Biochemistry \& Molecular Biology, Monash University, Clayton, Victoria, Australia, 2 The Ritchie Centre, Monash Institute of Medical Research, Clayton, Victoria, Australia, 3 Department of Medicine, University of Patras, Patras, Greece

\begin{abstract}
The CAMP response element binding protein 1 (Creb1) transcription factor regulates cellular gene expression in response to elevated levels of intracellular cAMP. Creb1 $1^{-1-}$ fetal mice are phenotypically smaller than wildtype littermates, predominantly die in utero and do not survive after birth due to respiratory failure. We have further investigated the respiratory defect of $\mathrm{Creb}^{-/-}$fetal mice during development. Lungs of $\mathrm{Creb} 1^{-1-}$ fetal mice were pale in colour and smaller than wildtype controls in proportion to their reduced body size. Creb1 ${ }^{-1-}$ lungs also did not mature morphologically beyond E16.5 with little or no expansion of airway luminal spaces, a phenotype also observed with the Creb $1^{-1-}$ lung on a $\mathrm{Crem}^{-1-}$ genetic background. Creb1 was highly expressed throughout the lung at all stages examined, however activation of Creb1 was detected primarily in distal lung epithelium. Cell differentiation of E17.5 $\mathrm{Creb} 1^{-/-}$lung distal epithelium was analysed by electron microscopy and showed markedly reduced numbers of type-I and type-Il alveolar epithelial cells. Furthermore, immunomarkers for specific lineages of proximal epithelium including ciliated, non-ciliated (Clara), and neuroendocrine cells showed delayed onset of expression in the Creb1 ${ }^{-1-}$ lung. Finally, gene expression analyses of the E17.5 $\mathrm{Creb}^{-1-}$ lung using whole genome microarray and qPCR collectively identified respiratory marker gene profiles and provide potential novel Creb1-regulated genes. Together, these results demonstrate a crucial role for Creb1 activity for the development and differentiation of the conducting and distal lung epithelium.
\end{abstract}

Citation: Bird AD, Flecknoe SJ, Tan KH, Olsson PF, Antony N, et al. (2011) cAMP Response Element Binding Protein Is Required for Differentiation of Respiratory Epithelium during Murine Development. PLoS ONE 6(3): e17843. doi:10.1371/journal.pone.0017843

Editor: Saverio Bellusci, Children's Hospital Los Angeles, United States of America

Received November 29, 2010; Accepted February 12, 2011; Published March 8, 2011

Copyright: ( 2011 Bird et al. This is an open-access article distributed under the terms of the Creative Commons Attribution License, which permits unrestricted use, distribution, and reproduction in any medium, provided the original author and source are credited.

Funding: This work was supported by a program grant of the National Health and Medical Research Council of Australia (ID no. 384100). The funders had no role in study design, data collection and analysis, decision to publish, or preparation of the manuscript.

Competing Interests: The authors have declared that no competing interests exist.

*E-mail: daniel.bird@monash.edu

\section{Introduction}

Survival at birth is critically dependent upon the ability of the lung to immediately take over the role of gas exchange, which in turn, is dependent upon the development of a mature respiratory system during the fetal period. The continuously branching terminal buds of the developing airways contain a population of multipotent epithelial stem or progenitor cells that give rise to the major cell types of the mature airway epithelium [1,2]. Neuroepithelial, ciliated, and finally non-ciliated secretory Clara cells differentiate within the airway buds that undergo branching morphogenesis during the pseudoglandular stage ( $\sim$ E10.5-E16.5), while more-distally located alveolar epithelial cells (AECs) begin to differentiate later during the canalicular stage (E16.5-E17.5). Correct differentiation into these cell types, particularly those populating the distal epithelium, is essential to ensure sufficient levels of pulmonary surfactant and surface area for gas exchange, which together are required for survival at birth. The molecular mechanisms regulating differentiation of the lung epithelium into its specialised cell types during development are only partially understood, however it is clear that specific nuclear transcription factors, often acting in complex regulatory networks, play a critical role in mediating this process [3]. In this report we have investigated the role of Crebl (cyclic adenosine 3',5'-monophosphate (cAMP) response element binding protein), a downstream transcriptional mediator for a range of systemic signalling factors, in respiratory epithelial differentiation.

Crebl is a member of the Creb/Atf subfamily of cAMPresponsive basic region-leucine zipper (bZIP) transcription factors which include activating transcription factor 1 (Atfl) and the cAMP response element modulatory protein (Crem) [4]. A large range of signalling factors including steroid hormones, peptide hormones, growth factors and other cytokines can induce activation of Crebl, usually via stimulation of pathways which increase intracellular levels of cAMP. Elevated levels of cAMP activate and release catalytic subunits of protein kinase A (PKA) which phosphorylate Crebl primarily at Ser133 [5,6,7]. Once phosphorylated, Crebl can bind as homo- or heterodimers with other Creb/Atf subfamily members to cAMP response elements or CREs ('TGACGTCA') in promoter regions and transactivate specific target genes [4].

Gene targeted mutations of the Creb/Atfl subfamily in mice have provided important insight into the biological function of these factors during development. Mice lacking Atf1 and Crem 
develop normally, although $\mathrm{Crem}^{-1-}$ males are sterile due to defective spermatogenesis [8,9]. Inactivation of Creb1 isoforms $\alpha$ and $\Delta\left(C r e b 1^{\alpha \Delta}\right)$ produce normal, viable mutant offspring, however these mice are found at a reduced mendelian frequency at birth suggesting a developmental disadvantage in utero [10]. On the other hand, combined deletion of Creb1 isoforms $\alpha, \beta$ and $\Delta$ $\left(\mathrm{Creb1}^{-/-}\right)$causes a complete loss of Creb1 function, leading to perinatal death [11]. Investigation of Creb/Atfl subfamily mouse mutants has also revealed that Creb/Atfl subfamily members may compensate for one another. Upregulation of Crem expression has been noted in many organs of $\mathrm{Creb}^{\alpha \Delta}$ and $\mathrm{Creb}^{-/-}$mice, particularly in the brain $[10,11,12]$. Furthermore, Atf1 ${ }^{-/-} \mathrm{Creb1}^{-/-}$ and $\mathrm{Atfl}^{+/-} \mathrm{Creb1}^{-/-}$double transgenic mice die early in development indicating that Crebl and Atfl may also compensate for each other [9].

The role of the Creb/Atfl subfamily in lung development however has not been well described. To further investigate the role of Crebl in the developing lung, we analysed the lung phenotype of Creb1 ${ }^{-1-}$ fetal mice in detail from E15.5 till just before birth at E18.5. We firstly show that Creb is required for morphological development of the lung beyond E16.5. Importantly, we also demonstrate that any possible compensatory effect caused by upregulation of Crem in the absence of Crebl does not alter the Creb $1^{-/-}$ lung phenotype. We found that although Creb is widely expressed in the lung it is activated primarily in the distal epithelium. Given this, we investigated whether differentiation of cells within the distal epithelium was adversely affected. Differentiation of cell types from more proximal conducting airway epithelium such as non-ciliated (Clara), ciliated and neuroendocrine lineages was also examined in the Creb1 $1^{-1-}$ lung. Finally, gene expression analyses of E17.5 $\mathrm{Creb1}^{-/-}$lungs using whole genome microarray and quantitative PGR (qPGR) was used to identify respiratory marker gene profiles in order to provide potential novel Creb1-regulated genes. Together, our results point toward a crucial role for Creblmediated signalling in the differentiation of both the developing distal and proximal lung epithelium.

\section{Materials and Methods}

Mice

All animal experimentation was approved by the School of Biomedical Sciences Animal Ethics Committee, Monash University (Ethics no. 2009/63), and was carried out according to the National Health and Medical Research Council of Australia guidelines for the breeding, care and use of genetically modified and cloned animals for scientific purposes 2007. Creb $1^{\text {lox } \mathrm{P} / \text { lox } \mathrm{P}}$ mice on a $\mathrm{C} 57 \mathrm{Bl} / 6$ genetic background were generated by genetargeting as described previously [12]. These mice were bred with mice harbouring a neural progenitor specific- (nestin) driven Cre recombinase to produce Creb1 $1^{\text {Nescre }}$ mice. A small proportion of the Creb1 $1^{\text {Nescre }}$ mice showed unexpected Cre activity in germline cells, which led to the production of fully heterozygous Creb1 $1^{+/-}$mice where the mutated allele contained a Cre-mediated deletion of exon 10 of the mouse Creb1 gene [12]. Timed-mated Creb1 ${ }^{+/-}$ mice were sacrificed at E15.5 to E18.5 p.c. (post coitum) by cervical dislocation for subsequent collection of fetal lungs. Timed matings of $\mathrm{Creb}^{+/-}$mice on a $\mathrm{Crem}^{-/-}$genetic background were also peformed to obtain $\mathrm{Creb1}^{-/-}, \mathrm{Crem}^{-{ }^{-}-}$double transgenic fetal mice. Tail snips were genotyped at the Creb1 and/or Crem gene locus by PGR.

\section{Histology and immunohistochemistry}

Lung tissue was fixed in $4 \%$ paraformaldehyde overnight, processed for paraffin embedding, and $5 \mu \mathrm{m}$ paraffin sections prepared for analysis. Sections were incubated at $60^{\circ} \mathrm{C}$ for 2 hours and then deparaffinised using three washes of xylene, hydrated with 3 washes of $100 \%$ ethanol, then tap water and stained with haematoxylin/eosin for histological analyses. For immunohistochemical analyses, sections were hydrated using several washes of $100 \%, 95 \%$ and $70 \%$ ethanol and finally $\mathrm{dH}_{2} \mathrm{O}$. For immunostaining procedures, antigen retrieval was performed by microwaving slides in $10 \mathrm{mM}$ sodium citrate for 20 minutes. Endogenous peroxidases were quenched with $3 \% \mathrm{H}_{2} \mathrm{O}_{2}$ in methanol. Sections were treated with either specific animal serum or $2 \%$ Bovine Serum Albumin (BSA) to block non-specific binding and incubated with the following primary antibodies: Ki67 (Labvision, Fremont, CA), ProSPC (Chemicon, Temecula, CA), Sox9 (kindly provided by Ass. Prof Vince Harley, Prince Henry's Institute, Melbourne), SPD (Abcam, Cambridge, UK), Scgblal (Santa Cruz, CA), Crebl (Cell Signaling, Danvers, MA) pCrebl (Cell Signaling), CGRP (Calibiochem, La Jolla, CA), FoxJ1 (ABR, Golden, CO), CD31 (Abcam), aSMA (Abcam), washed 3 times in PBST (PBS with $0.1 \%$ Tween-20) then incubated with biotinylated secondary antibodies, (either rabbit anti-goat, Zymed, San Francisco, CA, goat anti-rabbit, Vector laboratories, Burlingame, CA or rat anti-mouse IgG1, Invitrogen, Carlsbad, CA). In the case of SPD, tryamide signal amplification (Perkin Elmer, Waltham, MA) was used to enhance signal strength. Sections were again washed in PBST and the biotinylated secondary antibody detected using LSAB ${ }^{\circledR} 2$ Streptavidin-HRP (Dako, Glostrup, Denmark) and diaminobenzidine (DAB) (Dako) systems as per manufacturer's instructions and finally counterstained with haematoxylin. Sections were also treated with antibody diluent (no primary antibody) and secondary antibody to serve as a negative control.

\section{Cell proliferation index and TUNEL analysis}

Sections immunostained with Ki67/haematoxylin (as described above) were viewed using a light microscope and images from proximal and distal regions of the fetal lung from three E18.5 $\mathrm{Creb}^{-/-}$and three E18.5 wildtype mice were collected. For cell counting analyses, at least 1000 cells were counted per animal using multiple sections and fields of view per section. Fields of view were carefully chosen to avoid counting the same cell twice or more. Numbers of immuno-positive cells (brown from HRP-DAB reaction) were compared to the number of immuno-negative cells (purple from haematoxylin histological stain) to determine a labeling index. Terminal deoxynucleotidyl transferase-mediated dUTP-biotin nick end-labelling (TUNEL) was performed using an ApopTag Plus Peroxidase In Situ Detection Kit (Chemicon International, Temecula, CA) as per manufacturer's instructions. Briefly, hydrated fetal lung sections (see above) were treated with Proteinase K $(20 \mathrm{~g} / \mathrm{mL})$, washed in distilled water, and then treated with $3 \%$ hydrogen peroxide to quench endogenous peroxidases. The sections were then washed again in distilled water, treated with terminal deoxynucleotidyl transferase, before the reaction was stopped by incubation in stop/wash buffer. Sections were washed in PBS and then treated with antidigoxignenin conjugate at room temperature. After washing again in PBS, sections were treated with peroxidase substrate to visualize staining, washed in distilled water and counterstained with Nuclear Fast Red. Slides were dehydrated and mounted (as above).

\section{Isolation of total fetal lung RNA}

Total RNA was prepared from fetal lung by homogenization in TRIzol reagent (Gibco/BRL, Rockville, MD), a guanidinium isothiocyanate/phenol-based solution. Following chloroform extraction, RNA was precipitated with isopropanol, washed in $70 \%$ 
ethanol and redissolved in water. For microarray analysis, total RNA was further purified using the Purelink ${ }^{\mathrm{TM}}$ Micro-to-Midi ${ }^{\mathrm{TM}}$ Total RNA Purification System (Invitrogen).

\section{Synthesis of cDNA and Quantitative Real-Time PCR}

qPCR was performed using cDNA prepared from total lung RNA as described above and reverse transcribed into cDNA using random hexamers and M-MLV Reverse Transcriptase, RNase $\mathrm{H}$ Minus, Point Mutant (Promega, Madison WI). Oligonucleotide primer pairs for qPCR analyses were designed using the web-based Primer3 software. cDNAs were assayed in triplicate and levels of $18 \mathrm{~S}$ rRNA was used as a normalising control. Primer sequences were ( $5^{\prime}$ to $\left.3^{\prime}\right)$ : Atf1, forward, AGACGTACCAGATCGGTACCA, reverse, GTCATCACCACGGTCTGC; Crem, forward, GGAGGTCGGGTACGTAAAC, reverse, CTTCGATCGTGCTGTGATTC; Sftpa, forward, AATGGGAGTCGTCAGCTTG, reverse, ACTGACTGCCGATTGGTG; Sftpa, forward, CAGGTGCAGGTATCACGTC, reverse, GCTTTGGCACGAGAATTG; Sftpc, forward, GAGTCGACGGGATTACTCG, reverse, GATGAGAAGGCGTTTGAGG; Sftpd, forward, TCATGTGTAGCGCAACAGAG, reverse, AAACCTGGATCACCCTTCTC; Abca3, forward, ACTTGTATGCACAGCTGAAAGG, reverse, TTCGTCAGGACACTTCTGGA; Aqp5, forward, CTGCGGTGGTCATGAATC, reverse, CTACGCAGAAGACGCAGTGA; Foxj1, forward, AAGGCGACGAAGATCACTC, reverse, ATGGAATTCTGCGAGGTG; Scgb1a1, forward, GCTCAGGTTCTTCGGACA, reverse, CAGACTCTGATTCGATGAGGA; Calca, forward, GAGGGCTGTAGTGTCACTGC, reverse, GTTGTCCTTCACGACACGTC; Chi3l1, forward, GACGAGAAACACCAACGTGA, reverse, CGATCAAAGCGATAAGAACG; Lyz1/ 2, forward, GGTGACTGGGTGTGTTTAGC, reverse, TCGACGGTTGTAGTTTGTAGC; Lcn2, forward, GGAAATATGCACAGGTATCGTC, reverse, AAATACAATGGCGAACTGG; Hist2h3c1, forward, CTTGGGTTAGTTAACACGCTGA, reverse, TTCAAGATTGTCGTTCTAGATTGTTAC; Hist1h3g, forward, CTACGTCGTGGGTCTGTTTG, reverse, CAGAAACCCTTAAGCCGTCT; D6Mm5e, forward, TGTGCAAGTGAGAGGGAACT, reverse, GCTGGAGCACATCTTCTATT. Cycling was performed using Platinum ${ }^{\circledR}$ SYBR $^{\circledR}$ Green qPCR SuperMix (Invitrogen) on a Rotor-Gene ${ }^{\mathrm{TM}_{3000}}$ (Corbett Research, Sydney, NSW). qPCR data was analysed using RotorGene 6.0 software (Corbett Research, Sydney, Australia) and differential expression determined using the comparative delta-delta CT method [13].

\section{Analysis of AEC phenotypes}

For the purposes of transmission electron microscopy, the entire right lung of E17.5 $\mathrm{Creb1}^{-/-}(n=5)$ and wildtype $(n=4)$ fetal mice was post-fixed in $2 \%$ glutaraldehyde [14]. The lung was then cut into either two or more parts (depending on overall lung size), avoiding major airways and blood vessels. Tissue was washed in $0.1 \mathrm{M}$ cacodylate buffer, incubated in $2 \% \mathrm{OsO}_{4}$ (in $0.1 \mathrm{M}$ cacodylate buffer), dehydrated in a series of ethanol and propylene oxide washes and embedded in epoxy resin. Between two and three epoxy resin/tissue blocks were randomly chosen from each animal, ensuring that samples were taken from both the upper and lower regions of each lung. Ultra thin sections (70-90 nm) were cut using a diamond knife, stained with aqueous uranyl acetate and Reynolds lead citrate and coded for blinded analysis. Alveolar epithelial cells were identified using a transmission electron microscope (Joel $100 \mathrm{~s}$ ). For each animal, a minimum of 100 AECs were classified and the proportions of each phenotype determined by counting the number of nuclear profiles of each type $[14,15]$. Identification of AECs depended on clear visualiza- tion of the basement membrane with all AECs localised on the luminal surface of this membrane. AECs were categorized as one of four phenotypes; undifferentiated AECs, type-I AECs, type-II AECs and intermediate AECs according to strict morphological criteria. Undifferentiated AEGs were identified by their abundant cytoplasmic glycogen. Type-I AEGs had flattened cytoplasmic extensions, flattened nuclei, little perinuclear cytoplasm and few cytoplasmic organelles. Type-II AECs were rounded in shape with a rounded nucleus, had microvilli on their apical surface and abundant cytoplasmic organelles, including lamellar bodies. Numbers of lamellar bodies per cross-sectional area of each type-II AEC were also quantified. The intermediate cells were a heterogenous group that displayed characteristics of both type-I and type-II AECs; these cells have cytoplasmic extensions usually associated with type-I AEGs but also exhibit type-II AEC characteristics such as lamellar bodies and apical surface microvilli $[14,15,16,17,18]$.

\section{Microarray gene expression analysis}

Whole genome expression microarray analysis was performed by the Australian Genome Research Facility with total RNA from E17.5 $\mathrm{Creb1}^{-/-}$and wildtype fetal lung $(n=4) .3$ ug of RNA was subjected to rRNA removal procedure using RiboMinus Human/ Mouse Transcriptome Isolation Kit (Invitrogen) and subsequently labelled using the Affymetrix WT cDNA Amplification kit (Millenium Sciences). Single stranded cDNA was end-labelled using terminal deoxynucleotidyl transferase enzyme from the WT Terminal Labelling kit (Millenium Sciences). Labelled cDNA was then hybridised to the Affymetrix MouseExon 1.0 ST Array GeneChip (Millenium Sciences cat no. 900817). Chips were hybridized at $45^{\circ} \mathrm{C}$ for 16 hours, washed using the FS450_0001 (49 array format) fluidics script in the Fluidics Station 450 (Affymetrix), and scanned using the GeneChip Scanner 3000450 (Affymetrix). The scanner operating software, GCOS, converted the signal on the chip into a DAT file, which was used for generating CEL files for analysis. The affymetrix CEL files were imported into Partek ${ }^{\circledR}$ Genomics Suite software package, version 6.3, build 6.08.0205 Copyright (C) 2007 (Partek Inc., St. Louis, MO, USA.) and the analysis was performed using the predefined workflow for gene expression analysis. Expression values were background corrected using the GCRMA algorithm (Robust Multi-array analysis with correction for GC content) [19,20]. Data were normalized [21] and summarized using Median Polish algorithm. Principal Component Analysis was used to transform gene expression information into variance-based information. The normalized data were then subjected to ANOVA model using the Bonferroni method [22]. The False discovery rate (FDR) [23] was calculated based on the $p$-value $(\leq 0.01)$ from ANOVA. Genes that experienced a change of 1.5 fold or more in signal intensity and FDR of less than 0.05 were considered to be differentially expressed. The raw intensity data of each gene was used for the calculation of a "z-score". Z-scores for each gene were normalized and expressed as a unit of a standard deviation from the normalized mean of zero. All Affymetrix-microarray data presented in this manuscript is MIAME compliant and has been submitted to the European Bioinformatics Institute ArrayExpress repository, Cambridge, UK. The data can be viewed by accessing the following web site: http://www.ebi.ac.uk/microarray-as/aer/login and using the following information: Login: Reviewer_E-MEXP-1295, Password: 9achuSeC, Accession number E-MEXP-1295.

\section{Statistical Analysis}

The statistical significance of the cell proliferation index, qPCR expression profiles, AEC and lamellar body indexes were all determined by a non-paired Student's $t$-Test with statistical 
significance set at $p<0.05$ and error bars depicting standard error of the mean (SEM).

\section{Results}

\section{Morphological defects in lungs of $\mathrm{Creb}^{-1-}$ mice are first detected after E16.5}

Haematoxylin and eosin-stained fetal lung tissue sections of E15.5 to E18.5 $\mathrm{Creb1}^{-/-}$and wildtype mice were analysed for gross morphology using light microscopy. Lungs from E15.5 and E16.5 Creb1 $^{-1-}$ mice showed similar tissue morphology to wildtype with respect to proximal and distal airway structure (Fig. 1A-D). At E17.5 and E18.5, the lungs of wild type mice showed the expected progressive expansion of the terminal airsacs indicative of the normal transition from the pseudoglandular stage to the canalicular-saccular stages (Fig. 1E, G). In comparison, the lungs of E17.5 and E18.5 Creb1 ${ }^{-/-}$mice displayed little expansion of both the proximal and distal airways and showed a gross morphology comparable to E15.5-E16.5 lungs. The lack of round terminal saccular structures and the predominance of elongated tubules indicate an arrest or delay of normal lung development (Fig. $1 \mathrm{~F}, \mathrm{H}$ ). The proportion of $C \mathrm{Creb} 1^{-/-}$mice which were present in each litter at the stages collected was also determined. Creb1 $1^{-1-}$ mice were found at 12.7\% (E15.5), 9.0\% (E16.5), 6.7\% (E17.5) and $8.7 \%$ (E18.5) (Table S1). Interestingly, these proportions are lower than those found in a different strain of $\mathrm{Creb}^{-/-}$mice, which showed a $15 \%$ rate at birth [11].

Intact $\mathrm{Creb1}^{-/-}$and wildtype fetal mice and their lungs were also visualised at E17.5 when the developmental defect was first observed. Lungs from $\mathrm{Creb} 1^{-/-}$mice were paler in colour and noticeably smaller than wildtype although this was proportional to the smaller size $(30 \%)$ of the Creb1 $1^{-1-}$ pup (Fig. 1I). Creb/Atflmediated signalling has been strongly implicated as a regulator of both cell proliferation [24] and apoptosis [9] during development. Therefore we investigated whether a loss of cell proliferation or perhaps increased apoptosis was the cause of the reduction in size of the Creb1 $1^{-/-}$lung. Sections from E18.5 Creb1-/- and wildtype lungs were immunostained for Ki67 (Fig. 1J,K), a nuclear marker of cell division, and also assessed by a TUNEL analysis (Fig. 1M,N), which detects apoptotic nuclei. The average percentage of Ki67-postive cells however, was only slightly higher (1.2 fold, $p<0.05$, Fig. 1L) in the Creb $1^{-1-}$ lung relative to wildtype, indicating that lack of cell proliferation is not a contributing factor to the small size of the $C r e b 1^{-1-}$ lung. Apoptotic cells were detected at levels too small for a statistical analysis however there seemed to be no obvious differences between wildtype (Fig. 1M) and Creb1 $1^{-1-}$ (Fig. 1N) lung sections, indicating that increased apoptosis is also not a contributing factor toward the small size of the Creb1 $1^{-1-}$ lung.

\section{Compensatory up-regulation of Crem does not alter $\mathrm{Creb}^{-1-}$ morphology in the developing lung}

Previous findings have shown that expression of Crem is upregulated in many organs in the absence of Crebl [10], which may complicate the analysis of the $C r e b 1^{-/-}$phenotype. Using qPCR analysis, we found that levels of Crem and also Atf1 mRNA were increased 5.5 fold $(p<0.001)$ and 1.4 fold $(p<0.05)$ respectively in lungs of E17.5 $\mathrm{Creb}^{-/-}$mice $(n=4)$ relative to wildtype (Fig. 2A). We therefore examined lungs of E17.5 Creb1 ${ }^{-1-}$, $\mathrm{Crem}^{-1-}$ double transgenic mice and looked for potential morphological defects in addition to the already-described defects seen in the E17.5 $\mathrm{Creb1}^{-/-}$lung. In comparison to $\mathrm{Atf1} \mathrm{I}^{-/-}$, $\mathrm{Creb1}^{-/-}$and $\mathrm{Atf1^{+/- }}$, Creb1 $1^{-/-}$double transgenic mice [9], $\mathrm{Creb1}^{-/-}, \mathrm{Crem}^{+/-}$and $\mathrm{Creb1}^{-/-}, \mathrm{Crem}^{-/-}$double transgenic mice developed normally suggesting that Crebl and Crem do not act in concert to fulfil an essential role during embryogenesis (data not shown). Lungs from E17.5 $\mathrm{Creb1}^{-1-}, \mathrm{Crem}^{+/-}$(Fig. 2D) and $\mathrm{Creb1}^{-1-}, \mathrm{Crem}^{-1-}$ (Fig. 2E) mice also showed no overt morphological differences to the phenotype described for $\mathrm{Creb1}^{-/-}$, $\mathrm{Crem}^{+/+}$mice (Fig. 1F). To determine any differences in the lung caused by lack of Crem alone, we also analysed lung morphology in E17.5 $\mathrm{Crem}^{-1-}$ mice. As expected, lungs from $\mathrm{Crem}^{-1-}$ mice showed no obvious defects at E17.5 (Fig. 2C) in accordance with the normal postnatal survival phenotype of these mice [8]. Together, this suggests that the up-regulation of Crem in the lung of $\mathrm{Creb1}^{-/-}$mice has no effect on the respiratory phenotype.

\section{Creb1 is activated primarily in the distal lung epithelium during late gestation}

Using immunohistochemistry, we determined the localisation of Crebl protein at E16.5 and E18.5, timepoints which occur before and after the onset of the lung defect at E17.5 observed in $\mathrm{Creb}^{-1-}$ mice. Crebl protein was detected in the nuclei of most cells in the E16.5 and E18.5 wildtype lung. However, while epithelial cells from both proximal and distal lung almost always showed Crebl expression a large number of mesenchymal cells showed no Crebl immunoreactivity, particularly surrounding large airways (Fig. 3A, B). We also performed Crebl immunohistochemistry on sections of Creb $1^{-1-}$ lungs to validate our mouse knockout model. At E18.5, Crebl protein was strongly detected in wildtype lung (as previously mentioned), however in lung tissue of E18.5 $\mathrm{Creb1}^{-/-}$mice no Crebl immunoreactivity could be seen (Fig S1).

As the transcriptional activation of Crebl via cAMP is dependent upon specific phosphorylation at Ser133 [7], we analysed the presence of activated Crebl (pCrebl) using a phospho-Ser133 Crebl antibody. In E16.5 lung, pCrebl was detected sporadically in almost all cell types of the developing lung, however cells from the distal epithelium almost always showed pCrebl expression (Fig. 3C). In contrast, at E18.5 pCrebl expression in distal lung epithelium was much reduced, while more expression was seen in the interstitial mesenchyme (Fig. 3D). Increased levels of mesenchymal staining were also observed surrounding the proximal epithelium (Fig. 3D).

\section{Vascular development is unaffected in lungs of $\mathrm{Creb}^{-1-}$ mice}

Since we observed that intact lungs of $\mathrm{Creb}^{-/-}$fetal mice were paler than wildtype controls (Fig. 1I), and also that Crebl and pCrebl expression were often seen in pulmonary endothelial cells (data not shown), we reasoned that loss of Crebl may impair pulmonary circulation via improper development of the lung vasculature. To investigate this, lung sections of E16.5 to E18.5 wildtype and $\mathrm{Creb1}^{-{ }^{-}}$mice were stained for the endothelial marker CD31 (also known as Pecaml) using immunohistochemistry. In wildtype mice, CD31 staining showed the expected pattern of pulmonary vasculature development throughout the lung with normal appearance of large blood vessels and an extensive capillary network surrounding the distal epithelium (Fig. 4A,C,E). In lungs of E16.5 $\mathrm{Creb}^{-9-}$ mice, the vasculature structure appeared similar to E16.5 wildtype mice (Fig. 4B). At E17.5 and E18.5 the vasculature structure seemed disorganised in lungs of $\mathrm{Creb}^{-1-}$ mice relative to wildtype controls, and retained an immature morphology similar to E16.5 wildtype lungs (Fig. 4D,F). However it was also evident that the endothelial network remained intact in E17.5 and E18.5 Creb1 $1^{-/-}$lungs with evidence of large blood vessels and also capillaries in close proximity to the epithelium. We also stained wildtype and $\mathrm{Creb}^{-/-}$ 
E15.5
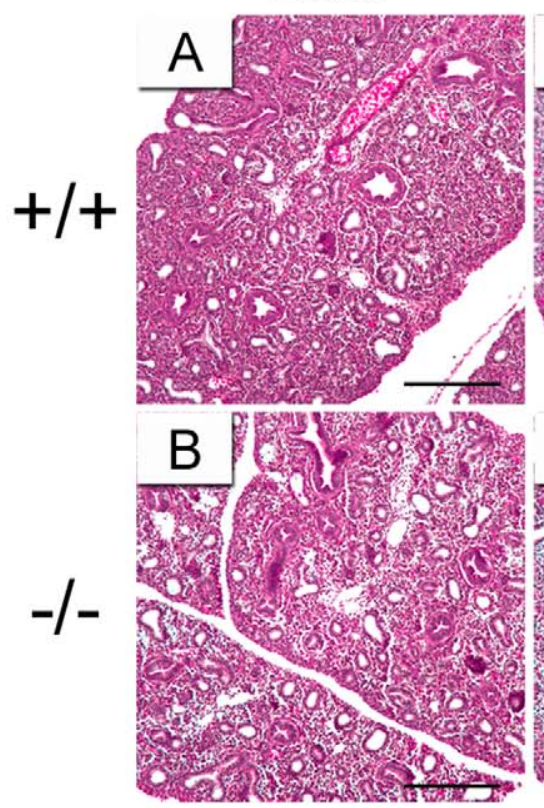

E16.5
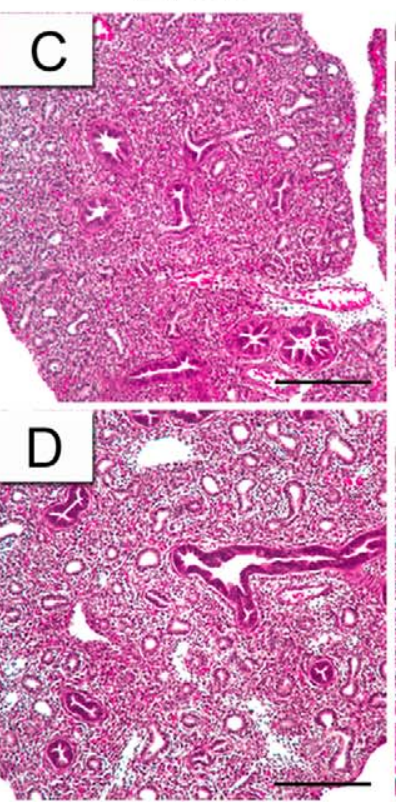

E17.5
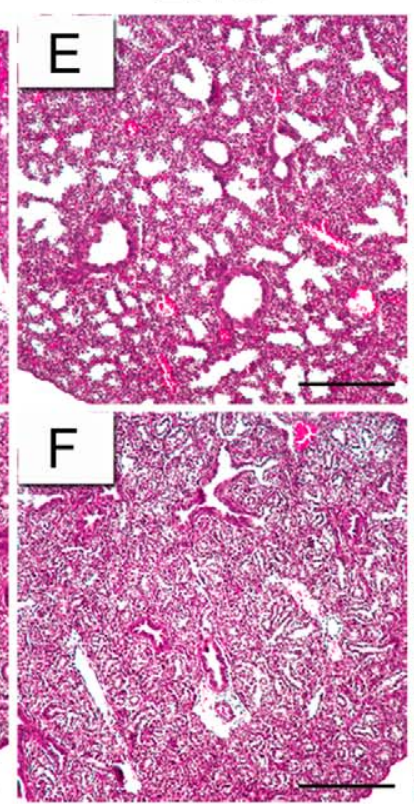

E18.5
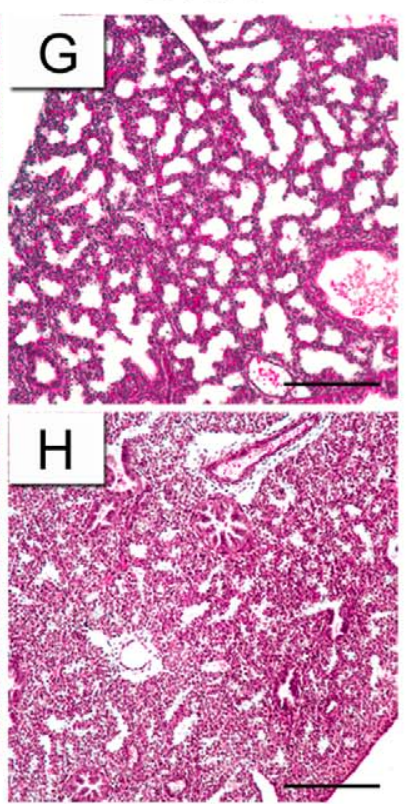

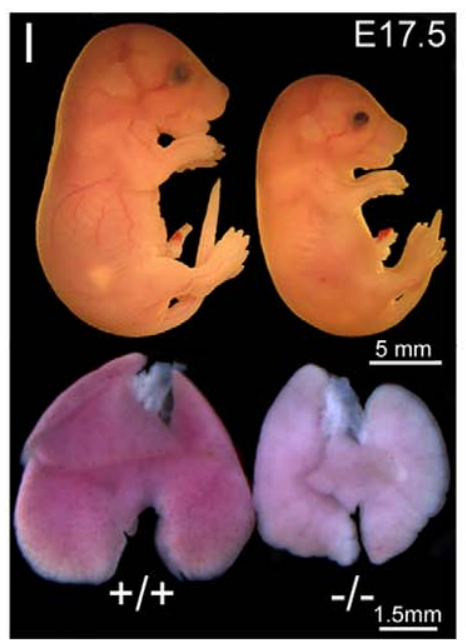

$\mathrm{L}$

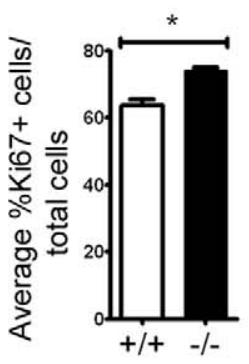

TUNEL
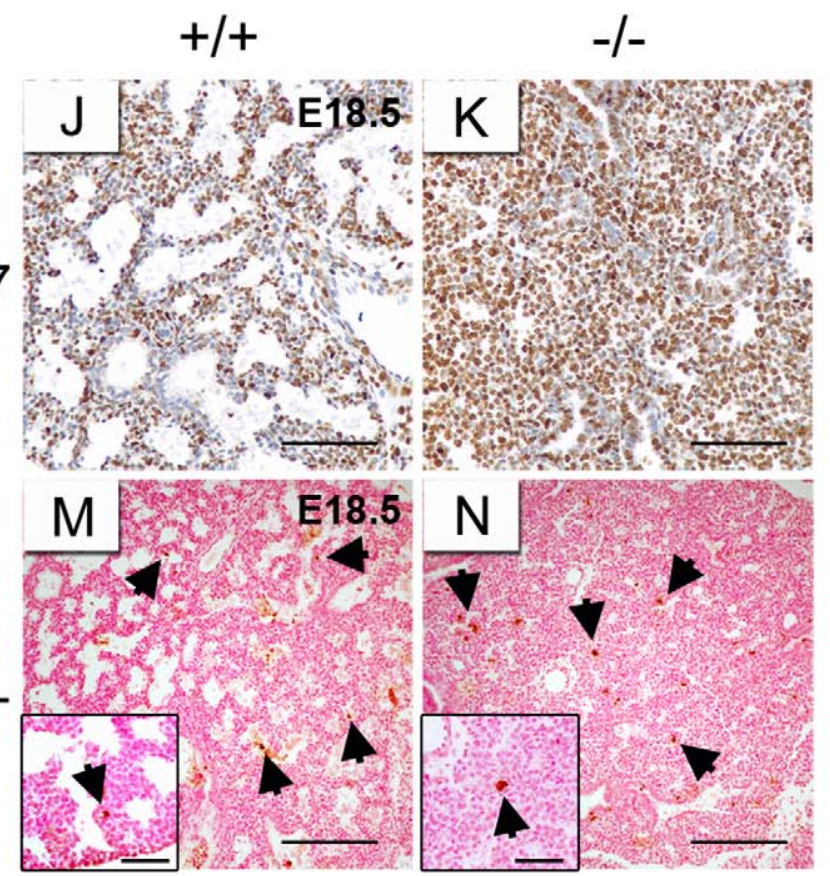

Figure 1. Morphological analysis of lung development in lungs of $\mathrm{Creb1}^{-1-}$ mice. Haematoxylin and eosin-stained tissue sections from E15.5-E18.5 Creb1 $1^{-1-}$ and wildtype lungs (A-H). Lung morphology of Creb $1^{-1-}$ and wildtype fetal mice was similar at E15.5 (A, B) and E16.5 (C, D). At E17.5 proximal and distal airways of Creb $1^{-1-}$ mice failed to expand and showed compacted tissue morphology (F). Lungs of E18.5 Creb $1^{-1-}$ mice showed a comparable morphology to $\mathrm{E} 17.5 \mathrm{Creb}^{-1-}$ lungs $(\mathrm{H})$. In comparison littermate controls at E17.5-E18.5 showed normal airway expansion (E, G). Lungs of E17.5 Creb1 ${ }^{-1-}$ mice were smaller than wildtype, though in approximate proportion to a reduced body size of Creb $1^{-1-}$ mice (I). Immunohistochemical analysis for the cell division marker Ki67 in lungs of E18.5 wildtype $(\mathrm{J})$ and $\mathrm{Creb}^{-{ }^{--}}$(K) mice. Quantification of Ki67-positive and -negative cells $(n=3)$ in total lung showed a small increase in cell proliferation in lungs of Creb1 $1^{-1-}$ mice at E18.5 (L). TUNEL analysis for apoptotic nuclei showed very rare, but comparable numbers of apoptotic cells in E18.5 wildtype (M) and $\mathrm{Creb}^{-1-}$ (N) lungs (arrows indicate apoptotic nuclei). Error bars represent SEM. Asterisk $(*)$ indicates $p<0.05$. Scale bars: $A-H, 200 \mu \mathrm{m} ; I, 5 \mathrm{~mm}$ for whole pups and $1.5 \mathrm{~mm}$ for fetal lungs as shown; J,K, $100 \mu \mathrm{m} ; M, N, 200 \mu \mathrm{m}$; insets for $M$ and $N, 20 \mu \mathrm{m}$. doi:10.1371/journal.pone.0017843.g001 
A

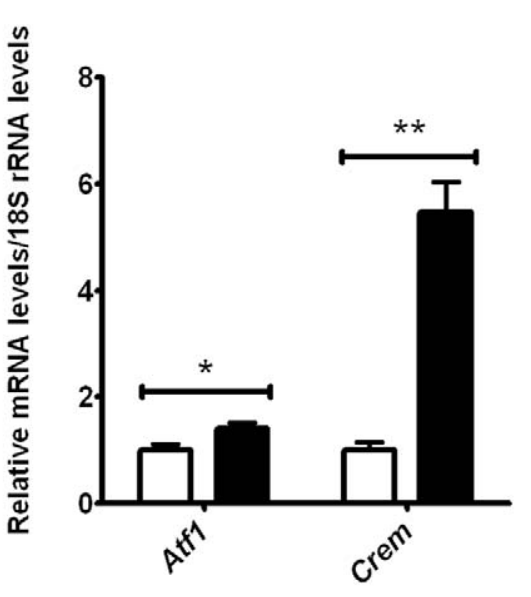

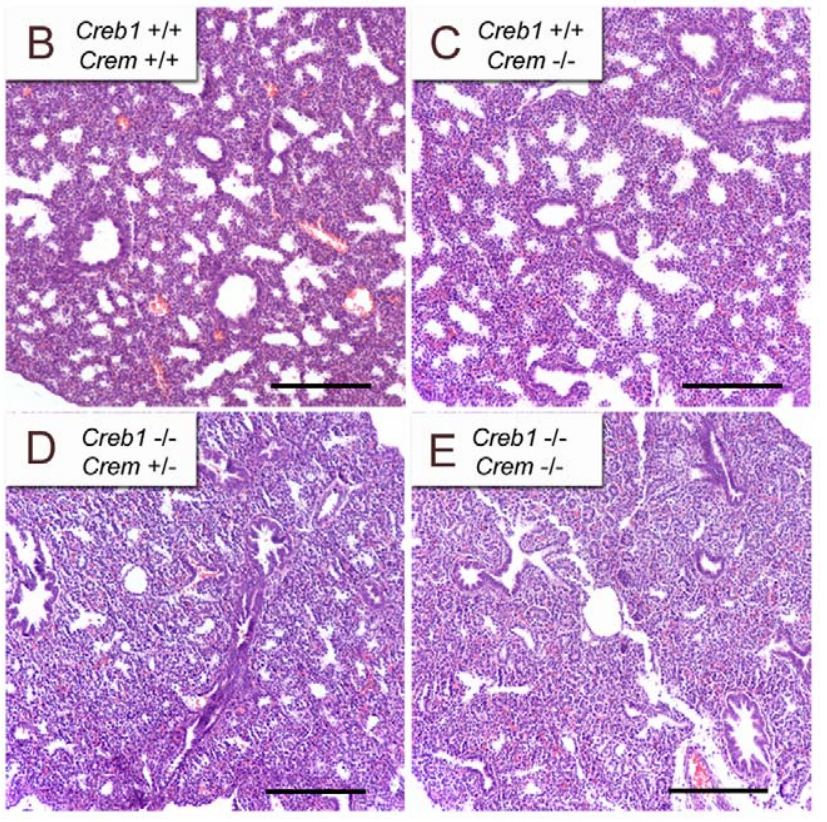

Figure 2. Compensatory up-regulation of $\mathrm{Crem}$ does not alter $\mathrm{Creb} 1^{-1-}$ morphology in the developing lung. $\mathrm{qPCR}$ analysis of $\mathrm{Crem}$ and Atf1 mRNA levels in the E17.5 $\mathrm{Creb}^{-1-}$ and wildtype lung $(n=4)(\mathrm{A})$. Haematoxylin and eosin-stained tissue sections from E17.5 lungs of $\mathrm{Creb}{ }^{+/+}$,

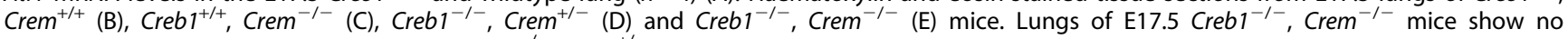
morphological differences as compared to $\mathrm{Creb}^{-1-}, \mathrm{Crem}^{+-}$mice. Error bars represent SEM. Asterisk (*) indicates $p<0.05^{\prime}$ and ${ }^{* *}$ indicates $p<0.001$. White bars: Wildtype, Black bars: Creb $1^{-1-}$. Scale bars: $100 \mu \mathrm{m}$.

doi:10.1371/journal.pone.0017843.g002

lung sections at E17.5 for the smooth muscle cell marker, alpha smooth muscle actin ( $\alpha \mathrm{SMA})$. In wildtype mice $\alpha \mathrm{SMA}$ staining showed a normal layer of smooth muscle surrounding large airways and blood vessels (Fig. 4G). Lungs of Creb1 ${ }^{-1-}$ mice also showed a normal layer of smooth muscle cells surrounding large airways and blood vessels (Fig. 4H). Together this suggests that lack of Crebl does not directly impair gross development of the pulmonary vasculature, although structural organisation of the microvasculature appears altered at E18.5.

\section{Defective AEC differentiation and lamellar body development in lungs of $\mathrm{Creb}^{-/-}$mice}

As activated Creb was found primarily in the distal lung epithelium, and that the onset of the $C r e b 1^{-1-}$ defect occurred at the developmental stage where AECs first begin to differentiate (E16.5-E17.5), we reasoned that an absence of Crebl would have an adverse effect on AEC differentiation. In order to investigate this, the lungs of E17.5 $\mathrm{Creb}^{-1-}(n=4)$ and wildtype $(n=5)$ mice were analysed using electron microscopy $(\mathrm{EM})$ and the proportions of undifferentiated, type-I, type-II and intermediate AECs (Fig. 5A) were determined using strict morphological criteria (see methods). Although the proportion of type-II AECs was significantly reduced 1.4 fold in the Creb1 $1^{-/-}$lung, proportions of type-I AEGs were most dramatically affected with a 19 fold reduction in the $C r e b 1^{-1-}$ lung relative to wildtype (Fig. 5B). In contrast, the proportion of undifferentiated AECs was significantly increased by 2.4 fold in the Creb1 $^{-1-}$ lung (Fig. 5B). The number of lamellar bodies per crosssectional area of type-II AECs was also significantly reduced by 1.9 fold in the Creb1 $1^{-1-}$ lung (Fig. 4J), suggesting that type-II AECs in the $\mathrm{Creb} 1^{-/-}$lung are relatively immature.

To further investigate AEC immaturity in $C r e b 1^{-1-}$ lungs we used qPCR to quantify mRNA levels of the surfactant protein genes: Sftpa, Sftpb, Sftpc and Sftpd, which are expressed within type-
II AECs (Fig. 5D). We also quantified mRNA levels for Abca3 (ATPbinding cassette sub-family A (ABC1), member 3) which encodes a factor required for lamellar body synthesis [25] as well as Aqp5 (Aquaporin 5), a marker for type-I AECs (Fig. 5D). Levels of surfactant protein gene mRNA showed little or no differences in the $\mathrm{Creb1} 1^{-1-}$ lung relative to wildtype, although the small reduction in $\operatorname{Sfpa}(1.7$ fold $)$ and $S f t p c$ (1.5 fold) did reach statistical significance. The only exception to this was Sftpd which showed a large (10.6 fold) reduction in mRNA levels. Levels of $A b c a 3$ mRNA were not significantly altered. qPCR analysis also found that $A q p 5$ mRNA levels were significantly reduced (8.5 fold) relative to wildtype, which is consistent with the large reduction in type-I AEC proportions we observed in the $\mathrm{Creb1^{-1- }}$ lung by EM analysis. These data suggest that differentiation of type-II AECs is mildly impaired in the Creb1 ${ }^{-/-}$lung, however differentiation of type-I AECs is completely blocked.

\section{Sox9 and ProSPC are highly expressed in the distal epithelium of $\mathrm{Creb}^{-/-}$lungs at late gestation}

We further verified that loss of Creb1 in the developing lung prevents normal differentiation of the distal epithelium. Sections of wildtype and $\mathrm{Crebl}^{-/-}$lungs were immunostained for the SRYbox containing gene 9 (Sox9) transcription factor, a marker of epithelial progenitor cells in the developing lung. Sox9 is expressed specifically in distal epithelial progenitor cells from E1 1.5 to E16.5, but is almost undetectable by E18.5 $[1,26]$. We detected low levels of Sox9 in wildtype lungs at E17.5 (Fig. 6A). However in E17.5 $\mathrm{Creb1}^{-/-}$lungs, the distal epithelial tubules were almost entirely populated by Sox9-positive cells (Fig. 6B). Additionally, we stained wildtype and Creb1 $1^{-1-}$ lung sections for ProSPC, which is expressed in the lung epithelium at approximately E11.5 until E17.5 whereupon it becomes restricted to type-II AECs [27]. As expected, we detected ProSPC only in type-II AEGs in E17.5 wildtype lung (Fig. 6C), however in E17.5 Creb1 $1^{-/-}$lungs, ProSPC 

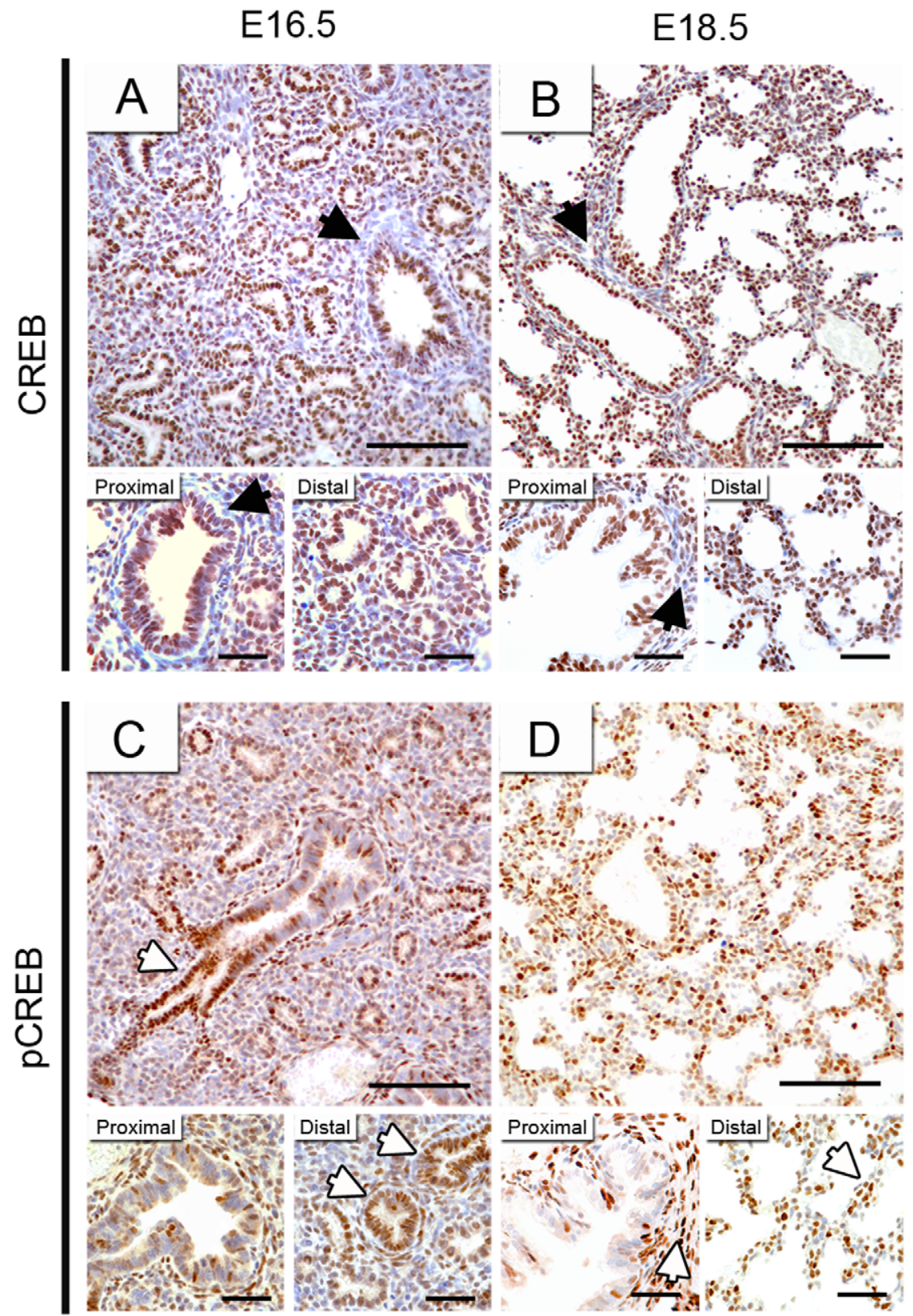

Figure 3. Creb1 is activated primarily in the distal lung epithelium during late gestation. Immunohistochemistry for Creb1 (A,B) and pCreb1 (C,D) on tissue sections of E16.5 and E18.5 wildtype lungs. Creb1 expression was detected in most cells at E16.5 (A) and E18.5 (B), though a proportion of mesenchymal cells surrounding large airways were Creb1-negative (Black arrows). At E16.5, pCreb1 staining was detected sparsely in the mesenchyme and proximal epithelium but in most cells of the distal epithelium (C, white arrows). At E18.5, pCreb1 staining was found primarily in the mesenchyme surrounding large airways and also within saccular walls ( $D$, white arrows). Scale bars: upper panels, $100 \mu \mathrm{m}$; magnified lower panels of proximal and distal lung, $25 \mu \mathrm{m}$.

doi:10.1371/journal.pone.0017843.g003

was found in almost all cells within distal epithelial tubules (Fig. 6D). Together, this suggests that the distal lung epithelium of $\mathrm{Creb}^{-1-}$ mice is almost entirely populated by undifferentiated progenitor cells at late gestation.

Sftpd is localised to the developing conducting airway epithelium, but is absent in the Creb1 $1^{-/-}$lung

As Sftpd mRNA levels were particularly reduced in the E17.5 Creb1 $^{-1-}$ lung compared with other surfactant protein genes, we investigated the localisation of Sftpd protein in wildtype lungs at E17.5 using immunohistochemistry and compared this to E17.5 $\mathrm{Creb1}^{-1-}$ lungs. We detected only modest expression of Sftpd in the cytoplasm of wildtype conducting airway epithelium (Fig. 7A), however no expression could be seen in any compartment of the Creb1 $1^{-/-}$lung (Fig. 7B). Interestingly, epidermal skin cells of the stratum spinosum layer showed strong Sftpd expression in E17.5 wildtype (Fig. 7G), but not E17.5 Creb1 ${ }^{-1-}$ (Fig. 7D) mice, suggesting that lack of Sftpd expression in $\mathrm{Creb1}^{-/-}$mice is not merely a secondary outcome of relative lung immaturity. 
CD31 $+/+$

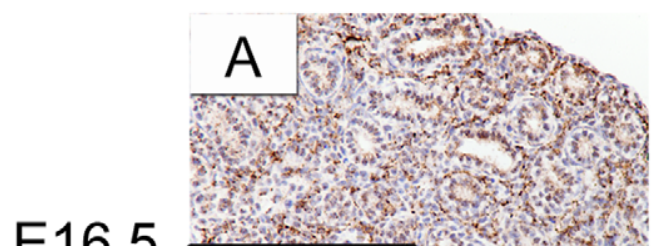

\section{E16.5}

E17.5

E18.5
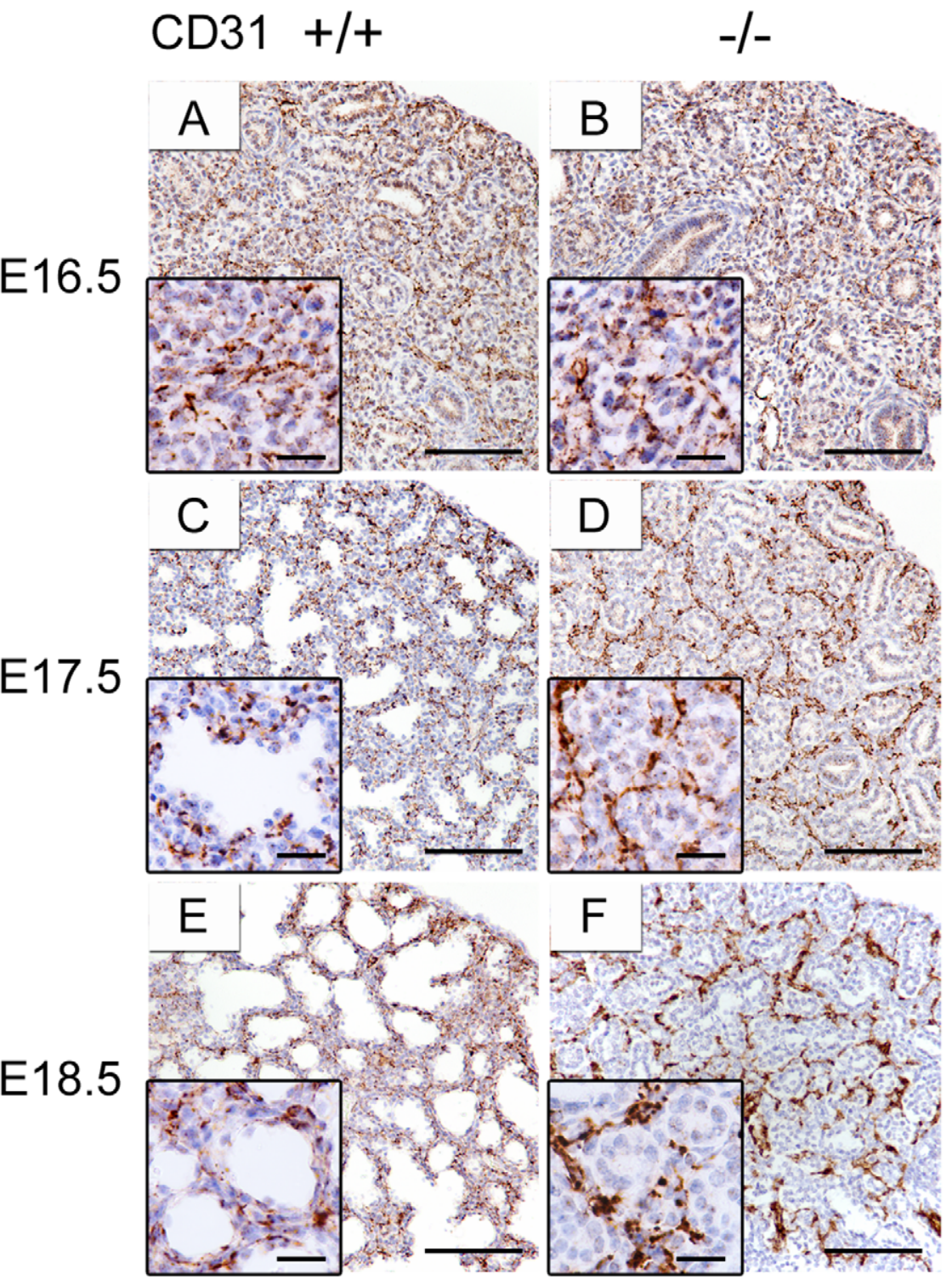

\section{aSMA}

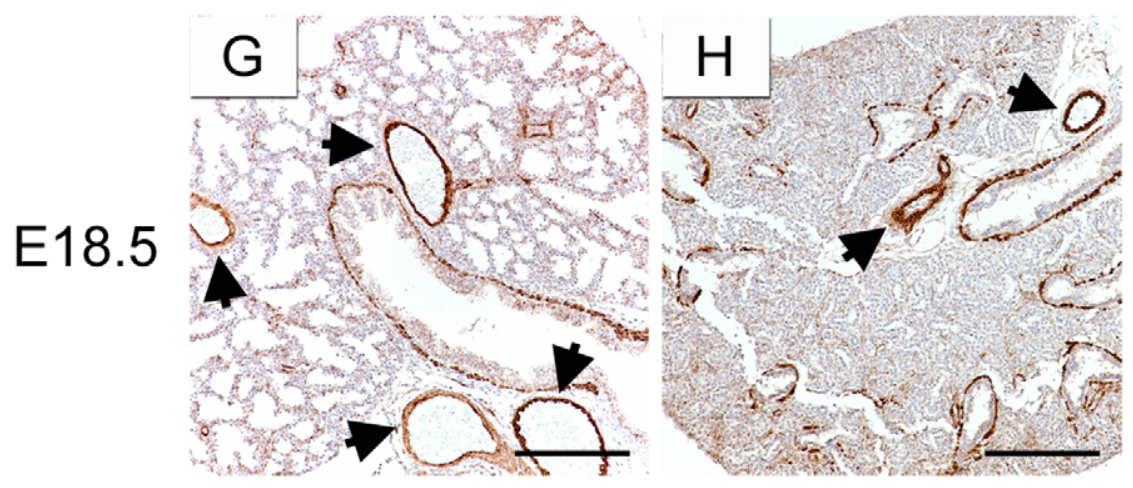

Figure 4. Vascular development is unaffected in lungs of $\mathrm{Creb}^{-{ }^{-1}}$ mice. Immunohistochemistry for CD31 (A-F) and $\alpha \mathrm{SMA}(\mathrm{G}, \mathrm{H})$ in the lung of E16.5 to E18.5 $\mathrm{Creb}^{-1-}$ and wildtype mice. In wildtype mice, CD31 immunostaining shows a normal vascular structure with large blood vessels and in capillaries in the distal lung $(A, C, E)$. Vascular structure in the lung of E16.5 Creb $1^{-1-}$ mice appears similar to E16.5 wildtype mice (B). An intact, although immature vasculature is seen in the lung of E17.5 (D) and E18.5 (F) Creb $1^{-1-}$ mice. A normal smooth muscle layer surrounding large blood vessels (arrows) was also detected in both E18.5 wildtype (G) and E18.5 Creb1 ${ }^{-1-}$ (H) mice. Scale bars: $100 \mu \mathrm{m}$; insets, $20 \mu \mathrm{m}$. doi:10.1371/journal.pone.0017843.g004 
A
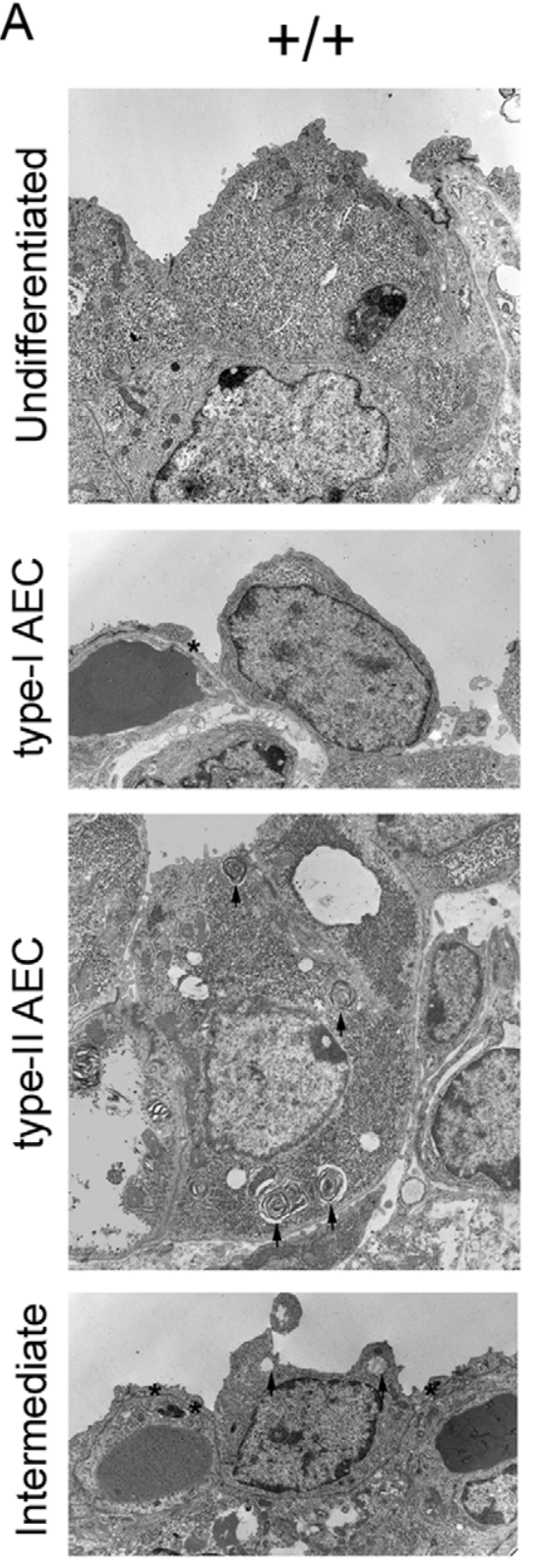

$-/-$
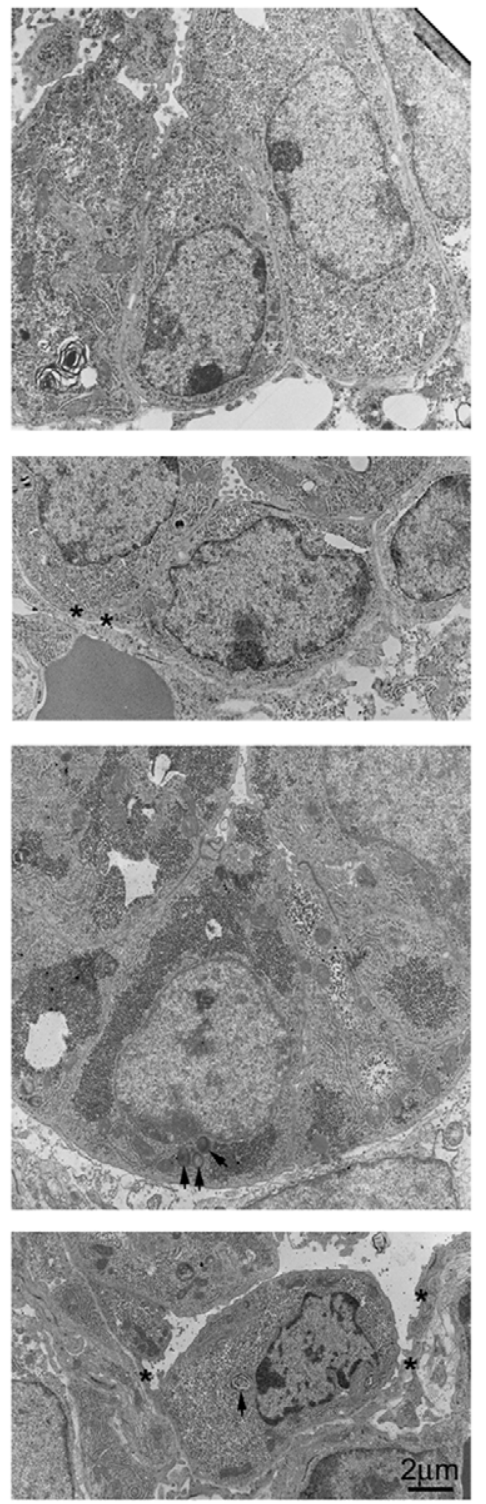

B
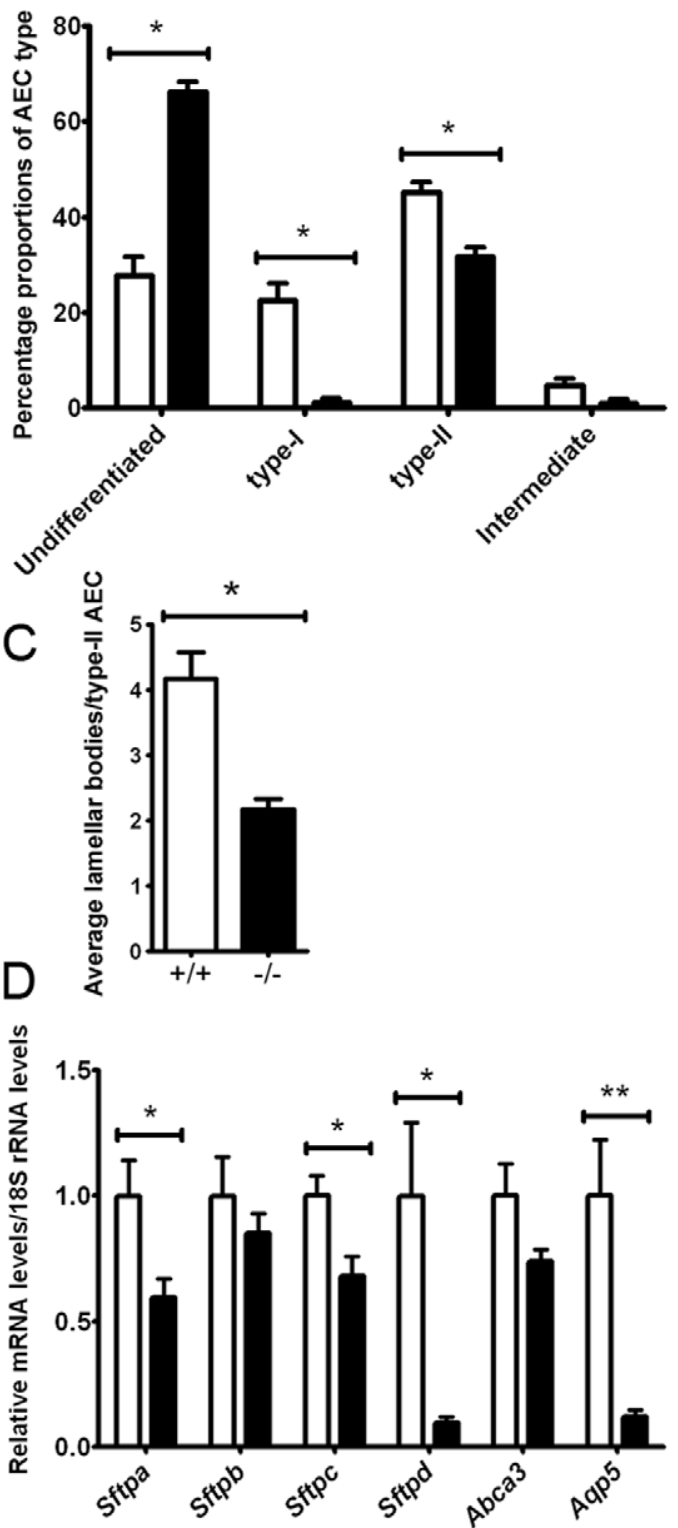

Figure 5. Defective AEC differentiation and lamellar body development in lungs of $\mathrm{Creb} 1^{-1-}$ mice. Tissue sections of E17.5 Creb $1^{-/-}$and wildtype lungs were analysed for AEC proportions by electron microscopy. Representative electron micrographs show the ultrastructural appearance of undifferentiated, type-l, type-II and intermediate AECs in wildtype and Creb1 ${ }^{-1-}$ mice (A). Asterisks indicate cytoplasmic projections of the developing type-I AECs whereas arrows indicate lamellar bodies in type-II AECs. Note that the lamellar bodies in the type-II AEC from wildtype mice are more numerous and more mature in appearance when compared to lamellar bodies in Creb $1^{-1-}$ mice. Quantification of relative AEC proportions showed a statistically significant reduction in proportions of type-II and type-I AECs, together with a statistically significant increase in undifferentiated AECs (B). Quantification of lamellar body number showed a statistically significant reduction per cross sectional area of type-II AEC in E17.5 $\mathrm{Creb1}^{-1-}(n=4)$ lungs relative to wildtype $(n=5)(C)$. qPCR analysis of mRNA levels for Sftpa, Sftpb, Sftpc, Sftpd and also Abca3 and Aqp5 in the lung of E17.5 $\mathrm{Creb}^{-1-}$ mice relative to wildtype $(n=4)$. Error bars represent SEM. Asterisk $\left(^{*}\right)$ indicates $p<0.05$ and ${ }^{* *}$ indicates $p<0.001$. White bars: Wildtype, Black bars: Creb1 $1^{-1-}$. Scale bars: $2 \mu \mathrm{m}$.

doi:10.1371/journal.pone.0017843.g005

Delayed expression of proximal epithelial cell markers in lungs of $\mathrm{Creb}^{-1-}$ fetal mice

Cell differentiation within the proximal airway epithelium was also examined in the lung of Creb $1^{-1-}$ fetal mice, using several specific immunomarkers of proximal airway epithelial cells. Scgblal (also known as CC10, CCSP) is a marker of non-ciliated secretory Clara cells, Foxjl (also known as HFH-4) is a marker of ciliated epithelial cells, and calcitonin gene related peptide (CGRP, also known as CALCA) is a marker of neuroepithelial cells. Using immunohistochemistry, lungs of E16.5 and E18.5 wildtype and Creb1 $1^{-1-}$ mice were analysed for expression of these markers as an indicator of proximal epithelial differentiation. Ciliated cells in the developing mouse lung first express Foxj1 at approximately E16.5 [28], and in lungs from E16.5 wildtype mice we detected a 


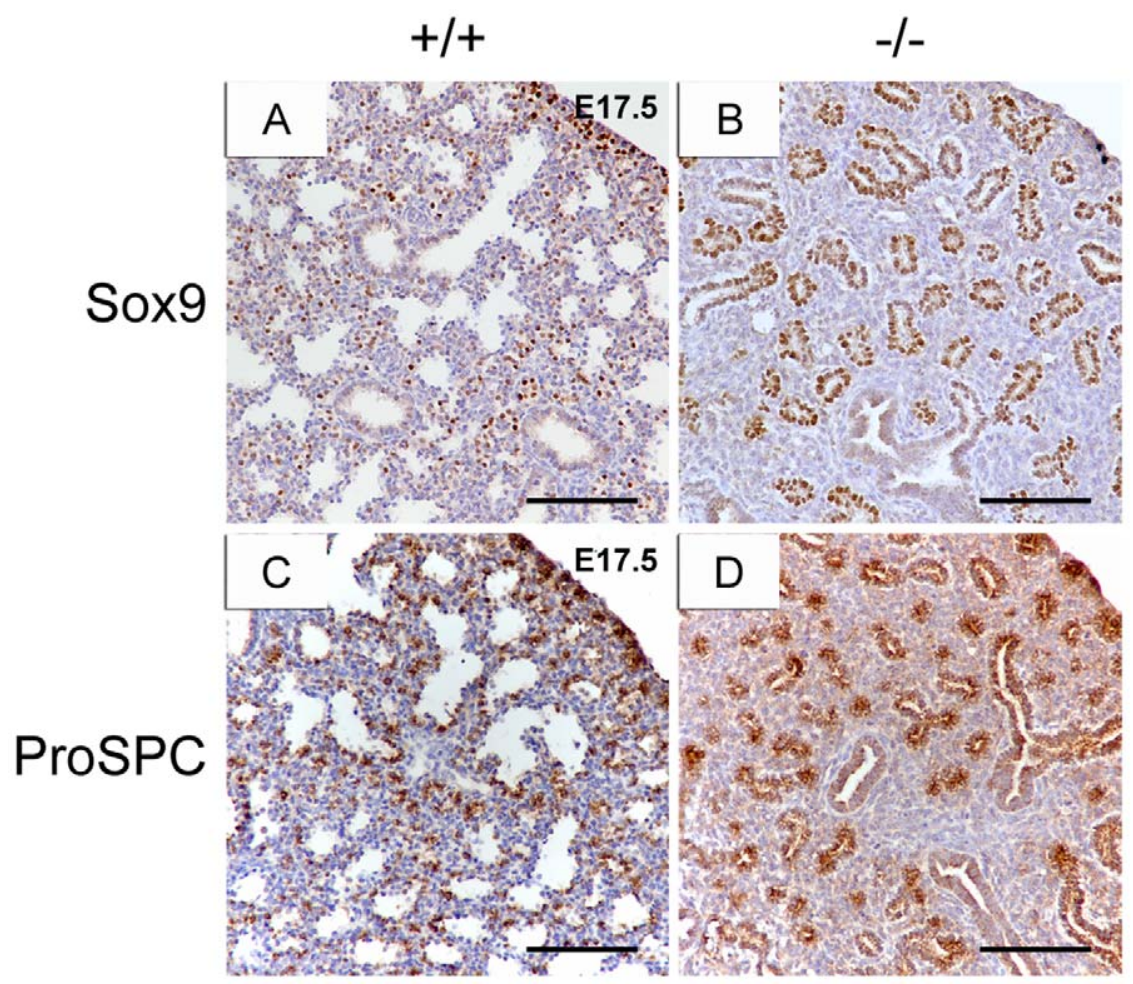

Figure 6. Sox9 and ProSPC are highly expressed in the distal lung epithelium of $\mathrm{Creb1}^{-1-}$ mice. Immunohistochemistry for the epithelial progenitor cell markers Sox9 (A,B) and ProSPC (C,D) in the lung of E17.5 Creb1 ${ }^{-1-}$ and wildtype mice. Sox9 was detected sporadically in distal epithelial cells of wildtype lungs (A), but was found within almost all cells of distal epithelial tubules in $\mathrm{Creb} 1^{-1-}$ lungs (B). In wildtype lungs, ProSPC was detected only in type-II AECs (C), however almost all cells of the distal epithelium showed strong ProSPC expression in Creb1 ${ }^{-1-}$ lungs (B). Scale bars: $100 \mu \mathrm{m}$.

doi:10.1371/journal.pone.0017843.g006
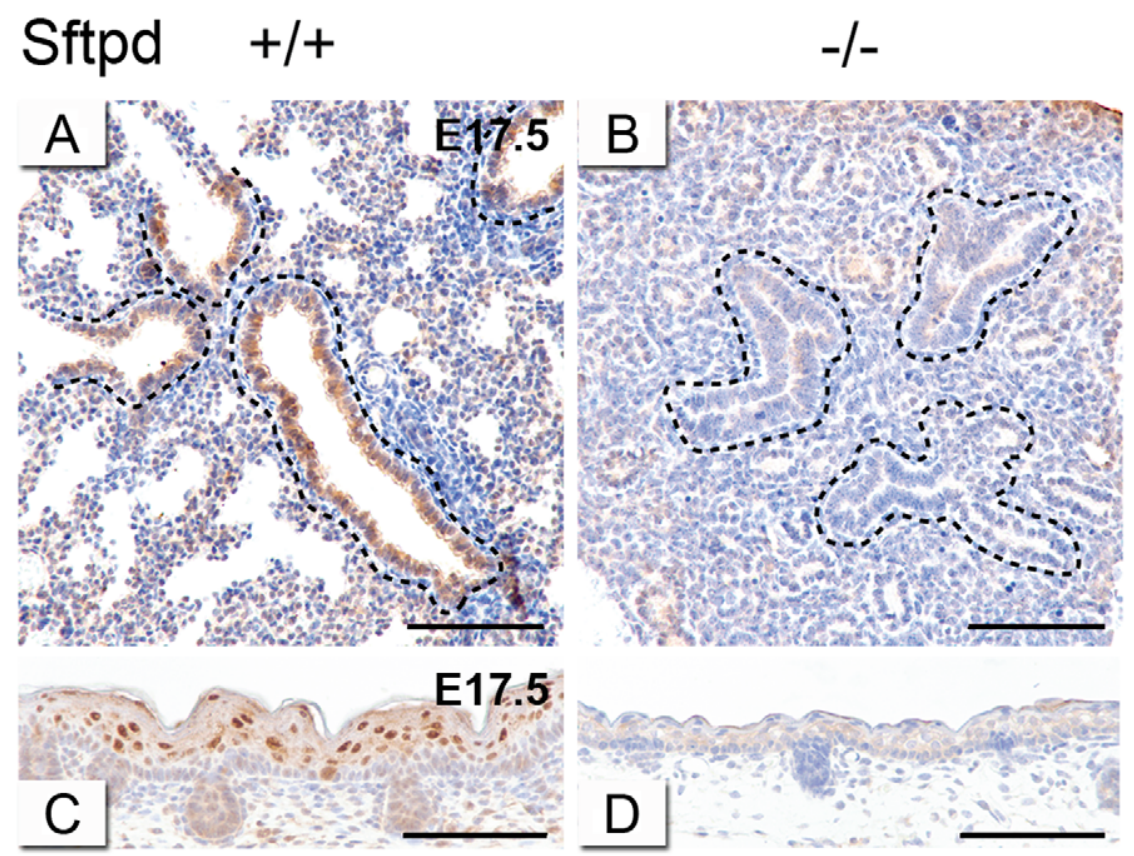

Figure 7. Sftpd is localised to the proximal airway epithelium, but is absent in the Creb1 ${ }^{-1-}$ lung. Immunohistochemistry for Sftpd protein in the lung $(A, B)$ and epidermis $(C, D)$ of E17.5 $\mathrm{Creb}^{-1-}$ and wildtype mice. Sftpd was localised primarily to the conducting airway epithelium (enclosed area) in the lung of wildtype mice (A), but was absent in the lung of $\mathrm{Creb}^{-1-}$ mice (B). Strong Sftpd expression was also detected in the stratum spinosum epidermal layer of wildtype (C), but not $\mathrm{Creb}^{-/-}$(D) mice. Scale bars: $100 \mu \mathrm{m}$. doi:10.1371/journal.pone.0017843.g007 
proportion of Foxj1-positive cells in the conducting epithelium (Fig. 8A) which increased in frequency at E18.5 (Fig. 8B). In the lung of $\mathrm{Creb1}^{-/-}$mice however, very few Foxj1-positive cells were detected at E16.5 (Fig. 8C), but were found at an increased frequency at E18.5 (Fig. 8D).

Clara cells first express Scgblal at approximately E17.5 in developing mouse lung [27]. Consistent with this, we did not detect Scgbla1-positive cells in E16.5 wildtype lung (Fig. 8E), but by E18.5 the majority of conducting airway epithelial cells expressed this marker (Fig. 8F). Lungs of Creb $1^{-1-}$ mice also showed no Scgblal-positive cells at E16.5 (Fig. 8G), however at E18.5 Scgbla1-positive cells were almost completely absent (Fig. 8H). CGRP is first detected at E16.5 in the mouse lung [29]. Similarly, in wildtype lungs we detected single CGRPpositive cells in conducting airway epithelium at E16.5 (Fig. 8I), and then CGRP-positive clusters in E18.5 (Fig. 8J), indicative of neuroendocrine bodies (clusters of neuroendocrine cells). In contrast, we could not detect GGRP-positive cells in lungs of E16.5 $\mathrm{Creb1}^{-/-}$mice (Fig. 8K), but by E18.5 neuroendocrine bodies were detected (Fig. 8L). Levels of Foxj1, Scgb1a1, and Calca mRNAs in the lung of E17.5 $\mathrm{Creb1}^{-1-}$ mice were also analysed using qPCR $(n=4)$ (Fig. 8M). Scgb1a1 mRNA levels were markedly reduced $\left(9.1\right.$ fold) in the Creb1 $1^{-1-}$ lung $(p<0.05)$ and Calca mRNA levels were also reduced $(2.4$ fold, $p<0.05)$. Foxf1 mRNA levels were reduced in the $C r e b 1^{-/-}$lung (1.6 fold) though did not quite reach statistical significance $(p=0.053)$. Together, this indicates pulmonary ciliated and neuroendocrine cell differentiation may be delayed in the Creb1 $1^{-1-}$ lung while Clara cell differentiation is severely disrupted.

\section{Microarray analysis identifies potential Creb1-regulated genes in the developing mouse lung}

To identify Crebl-regulated genes that may contribute to the observed defect at E17.5, total RNA from lungs of E17.5 wildtype and $C r e b 1^{-/-}$mice ( $n=4$ for each) was analysed using Affymetrix mouse whole-genome expression exon microarray slides. A list of 694 genes was identified as being differentially expressed in lungs of $\mathrm{Creb1}^{-/-}$mice relative to wildtype; 416 gene targets showing reduced mRNA levels, and 278 gene targets showing increased mRNA levels. Investigation of the top ten most differentially expressed targets in the microarray list (Table 1) identified a number of genes known or suspected to be over- or underexpressed in the lungs of $C r e b 1^{-1-}$ fetal mice. These included: Sftpd, which we found under-expressed in the lung of E17.5 Creb1 ${ }^{-1-}$ mice as previously described (Fig. 5D and Fig. 7), Scgb1a1, which we found did not show evidence of protein expression until E18.5 in the Creb1 ${ }^{-/-}$lung (Fig. 8), and Crem, which we found over-expressed in the lung of E17.5 Creb1 ${ }^{-\prime-}$ mice (Fig. 2A). Interestingly, a large proportion of the top ten most-highly under-expressed genes in the $\mathrm{Creb}^{-/-}$lung are associated with a role in host defence and inflammatory response (Chi3l1, Lyz1, Lcn2, Scgb1a1, Hc, Sftpd, Vnn1 and H2-10)(Table 1). Other highly under-expressed genes with known or predicted roles in immune response also appear with high frequency further down in our list including Ier3 $\left(12^{\text {th }}\right)$, Tnfaip $\left(14^{\text {th }}\right), H p\left(16^{\text {th }}\right)$, Lyz2 $\left(21^{\text {st }}\right)$ and $C 3\left(23^{\mathrm{rd}}\right)$. Over-expressed targets in the Creb1 $1^{-1-}$ lung however did not seem to belong to a particular ontology or function.

In order to validate our microarray data, we further investigated differential gene expression for the three most-highly downregulated (Chi3l1, Lyz1 and Lcn2) and up-regulated (Hist2h3c1, Hist $1 \mathrm{~h} 3 \mathrm{~g}$ and $\mathrm{D} 6 \mathrm{Mm} 5 \mathrm{e}$ ) gene targets on our list in the lung of E17.5 Creb1 $^{-{ }^{-}}$mice using qPGR $(n=4)$. Levels of Chi3l1 and Lcn2 mRNAs were reduced 12.5 fold $(p<0.001)$ and 2.9 fold $(p<0.05)$ in respectively (Fig. 9A). Due to very high sequence identity between $L y z 1$ and $L y z 2$ in mice, qPCR primers recognised $L y z 2$ as well as Lyz1, and we detected a 2.6 fold $(p<0.05)$ reduction in Lyz1/2 levels in the Creb1 $1^{-1-}$ lung (Fig. 8A). Levels of Hist $2 h 3 c 1$ mRNA were increased 4.0 fold in the Creb1 $1^{-/-}$lung, but did not quite reach statistical significance $(p=0.09)$, while Hist 1 h $3 g$ and D6Mm5e mRNA levels increased $5.0(p<0.05)$ and 6.5 fold $(p<0.05)$ respectively, in the $C r e b 1^{-/-}$lung (Fig. 9B). Taken together, these data support the validity of our microarray analysis.

\section{Discussion}

A detailed analysis of the respiratory phenotype in fetal $\mathrm{Creb1}^{-/-}$ mice has been performed and shows severely disrupted conducting and distal airway development, evident by E17.5. Furthermore, the timing of cell differentiation for a range of lung epithelial populations was strongly disrupted in lungs of $\mathrm{Creb1} 1^{-1-}$ mice.

We first show via Ki67 and TUNEL immunostains that the respiratory defect in the lung of $\mathrm{Crebl}^{-1-}$ mice is likely not correlated with a loss of cell proliferation or increased apoptosis, as might be expected from a lack of Crebl during embryonic development. For example, loss of Crebl specifically in chondrocytes reduces chondrocyte cell proliferation in mice at E14.5 to E17.5 [24] while loss of Crebl specifically in the brain on a $\mathrm{Crem}^{-/-}$ genetic background causes high levels of neuronal cell apoptosis at E18.5 [12]. Secondly, although we find that the lung from $\mathrm{Creb} 1^{-/-}$ mice are pale in colour, the respiratory defect we observe is unlikely to be due to impaired pulmonary circulation as we show that loss of Crebl did not seem to affect development of the lung vasculature. This is further supported by a previous report which found no abnormalities in mice lacking Crebl specifically in cardiomyocytes [30].

It is also important to note that the respiratory defect in $\mathrm{Creb} 1^{-/-}$ mice is unlikely to be merely a secondary outcome of general developmental retardation or arrest. For example, levels of surfactant protein mRNAs, with the exception of Sftpd, were not reduced in the lung of E17.5 Creb1 $1^{-/-}$mice, whereas it would be expected that surfactant protein gene expression between a normal and a developmentally arrested fetus would be highly divergent, particularly considering the rapid increase in surfactant protein gene expression following type-II AEC differentiation at E17.5 $[27,31]$. Additionally, a previous investigation indicated that the brain of E17.5 wildtype mice was clearly more developed than those of E18.5 Creb1 $1^{-/-}$mice, despite their comparable size [11]. Therefore we conclude that the respiratory phenotype in $C r e b 1^{-1-}$ mice is caused directly by a loss of Crebl-mediated activity intrinsically within the developing lung.

This phenotype was demonstrated most clearly in the distal epithelium at E17.5, with severely disrupted differentiation of AECs. Differentiation of type-II AECs during lung development can occur either from early distal epithelial progenitors or via trans-differentiation from type-I AECs [14,15]. In contrast, type-I AECs usually differentiate from a type-II AEC progenitor [32] but can also differentiate directly from distal epithelial progenitors as type-I AECs are often detected at an earlier developmental stage than type-II AECs $[14,33]$. As the proportion of undifferentiated AECs remained high at E17.5 in the Creb1 $1^{-/-}$lung, it is clear that Crebl is required for both type-I and -II AEC differentiation from undifferentiated distal lung epithelial progenitor cells, though not absolutely required for type-II AEC differentiation. On the other hand, Crebl function is essential for type-I AEC differentiation from both type-II AECs and undifferentiated distal epithelial progenitors. 


\section{E16.5}

Foxj1

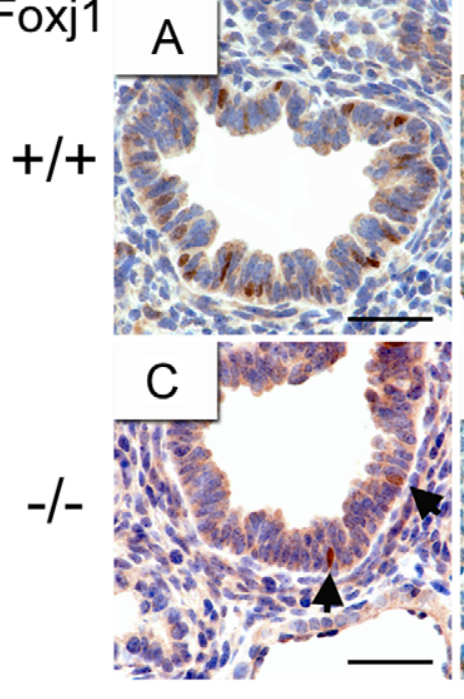

E16.5

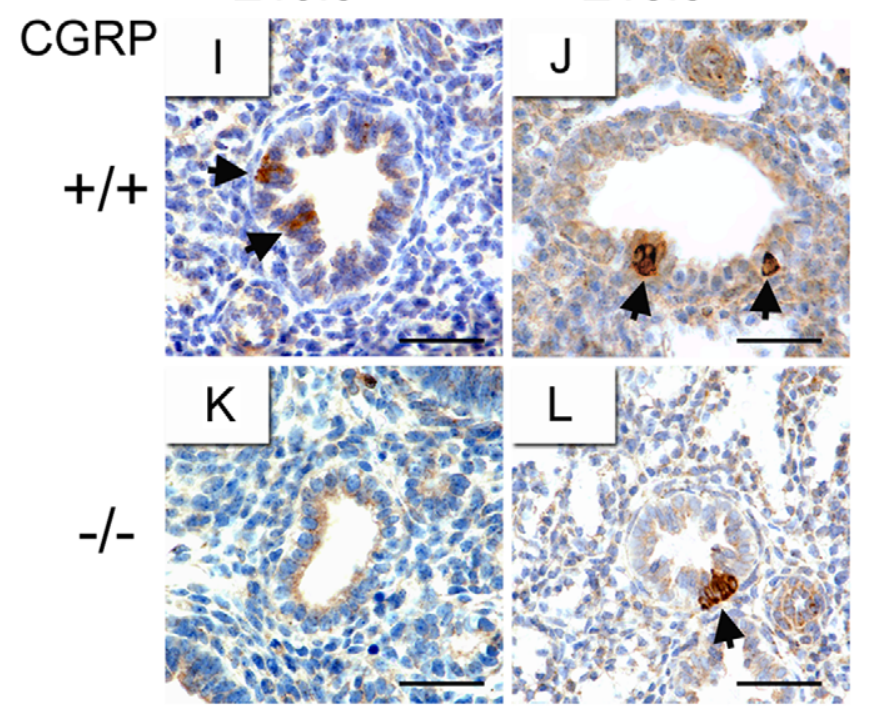

E16.5

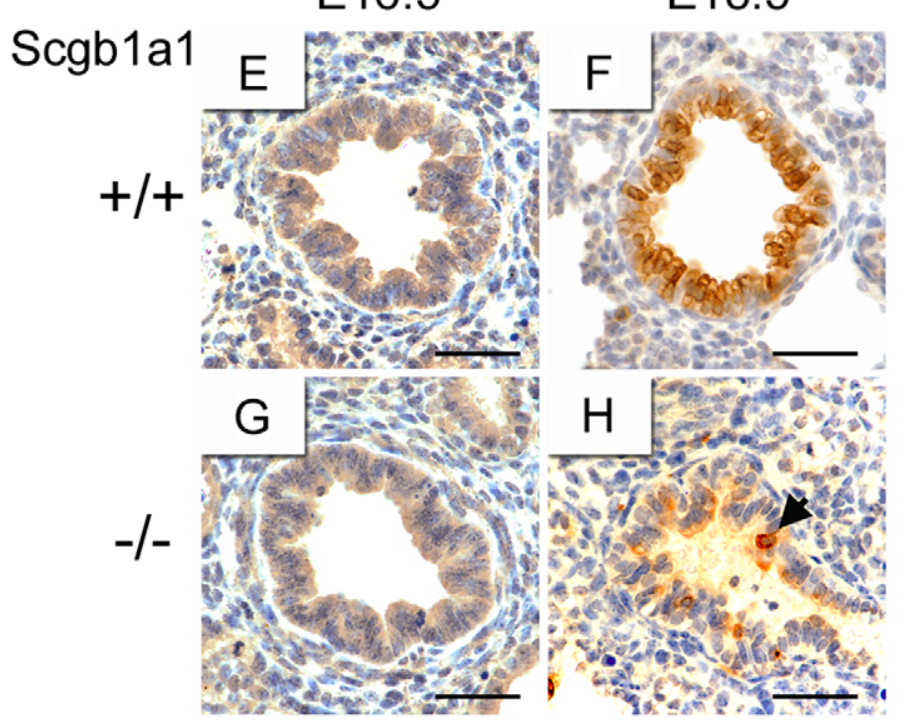

M

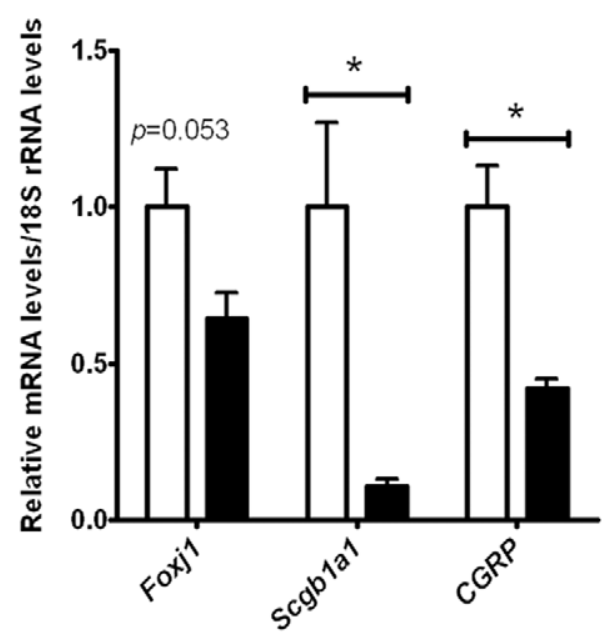

Figure 8. Delayed proximal epithelial cell differentiation in lungs of $\mathrm{Creb1}^{-1-}$ fetal mice. Tissue sections from the lung of E16.5 and E18.5 $\mathrm{Creb}^{-1-}$ and wildtype were immunostained for Foxj1 (A-D), CC10 (E-H), and CGRP (I-L). At E16.5 Foxj1-positive cells were readily detected in conducting airway epithelium (A), but much less frequently in $C r e b 1^{-1-}$ lungs ( $C$, arrowheads show two Foxj1-positive cells). Increased frequency of Foxj1-positive cells was detected in the lung of both E18.5 wildtype (B) and E18.5 Creb1 ${ }^{-1-}$ (D) mice. In wildtype lungs, Scgb1a1-positive cells were not detected at E16.5 (E,) but were common at E18.5 (F). In the lung of E18.5 Creb1 ${ }^{-/}$mice, Scgb1a1-positive cells were almost completely absent (H, arrowhead shows a solitary Scgb1a1-positive cell). Single CGRP-positive cells were detected at E16.5 in wildtype (I, arrowheads show CGRP-positive cells), but not $\mathrm{Creb}^{-1-}$ (K) lungs. At E18.5 Clusters of CGRP-positive cells were then detected in both wildtype and Creb ${ }^{-1-}$ lungs (J,L, arrowheads show CGRP-positive cell clusters). qPCR analysis of mRNA levels for Foxj1, Scgb1a1 and Calca in the lung of E17.5 Creb $1^{-1-}$ mice relative to wildtype $(n=4)(\mathrm{M})$. Error bars represent SEM. Asterisk $\left(^{*}\right)$ indicates $p<0.05$. White bars: Wildtype, Black bars: Creb $1^{-1-}$. Scale bars: $A-L, 50 \mu \mathrm{m}$. doi:10.1371/journal.pone.0017843.g008

We have also identified a role for Crebl in the timing of differentiation of the proximal lung epithelium. Immunomarkers for the ciliated (Foxj1), non-ciliated secretory Clara (Scgblal) and neuroendocrine (GGRP) cell populations, which together constitute the majority of the conducting airway epithelium, all show delayed appearance in the lung of Creb1 $1^{-/-}$mice. Crebl may therefore mediate differentiation throughout the entire conducting airway epithelium though this needs to shown for other epithelial populations such as goblet and basal cells, which appear in the upper bronchial and tracheal epithelium. It is also evident that increased differentiation of Foxj1-, Scgbla1-, and CGRP-positive cells continues to occur beyond E17.5 in lungs of $\mathrm{Creb}^{-/-}$mice when structural maturation of lungs seems to be arrested. This invites the possibility that other epithelial populations, such as AECs could further differentiate after E18.5 in the Creb1 $1^{-/-}$lung, however as Creb1 $1^{-1-}$ mice die at birth this is currently impossible to investigate.

The mechanism by which Crebl contributes to structural maturation or epithelial differentiation in the developing lung is 
Table 1. Differentially expressed genes in the lung of E17.5 $\mathrm{Creb} 1^{-/-}$mice identified by whole-genome microarray analysis.

\begin{tabular}{|c|c|c|c|c|}
\hline Accession No. & Gene name & Fold change & $p$ value & Function \\
\hline NM_007695 & Chitinase 3-like 1 (Chi3/1) & -41.3 & 0.0002 & Inflammatory response \\
\hline NM_013590 & Lysozyme 1 (Lyz1) & -16.3 & $>0.0001$ & Defense response \\
\hline NM_008491 & Lipocalin 2 (Lcn2) & -11.3 & 0.0031 & Inflammatory response \\
\hline NM_011681 & $\begin{array}{l}\text { Secretoglobin, family } 1 \mathrm{~A} \text {, member } 1 \text { (uteroglobin) } \\
\text { (Scgb1a1) }\end{array}$ & -10.9 & 0.0013 & Inflammatory response \\
\hline NM_010406 & Hemolytic complement $(H c)$ & -9.4 & 0.0002 & Inflammatory response \\
\hline NM_009160 & Surfactant associated protein D (Sftpd) & -8.9 & 0.0001 & Surfactant homeostasis \\
\hline NM_009127 & Stearoyl-Coenzyme A desaturase 1 (Scd1) & -8.5 & 0.0002 & Fatty acid biosynthesis \\
\hline NM_020509 & Resistin like alpha (Retnla) & -7.1 & 0.0001 & Regulation of apoptosis \\
\hline NM_011704 & Vanin $1($ Vnn1) & -6.3 & 0.0013 & Inflammatory response \\
\hline NM_010395 & Histocompatibility 2, T region locus 10 (H2-T10) & -5.6 & 0.0036 & Inflammatory response \\
\hline NM_207161 & cDNA sequence $B C 048355$ (BC048355) & 2.5 & 0.0008 & Unknown \\
\hline BC117501 & RIKEN cDNA F630043A04 gene (F630043A04Rik) & 2.5 & 0.0021 & Unknown \\
\hline NM_001110859 & cAMP responsive element modulator (Crem) & 2.7 & $>0.0001$ & Spermatogenesis \\
\hline NM_027664 & RIKEN cDNA 4933426K21 gene (4933426K21Rik) & 2.8 & $>0.0001$ & Unknown \\
\hline NM_021475 & ADAM-like, decysin 1 (Adamdec1) & 3.0 & $>0.0001$ & Integrin signalling pathway \\
\hline NM_175499 & SLIT and NTRK-like family, member 6 (Slitrk6) & 3.1 & 0.0007 & Axongenesis \\
\hline NM_010733 & leucine rich repeat protein 3, neuronal (Lrrn3) & 3.3 & 0.0003 & Endocytosis \\
\hline AF084364 & $\begin{array}{l}\text { DNA segment, Chr 6, Miriam Meisler 5, expressed } \\
(D 6 M m 5 e)\end{array}$ & 3.4 & 0.0001 & Gametogenesis \\
\hline NM_145073 & histone cluster 1, H3g (Hist1h3g) & 3.9 & 0.0037 & Nucleosome assembly \\
\hline NM_178216 & histone cluster 2, H3c1 (Hist2h3c1) & 4.8 & 0.0017 & Nucleosome assembly \\
\hline
\end{tabular}

The top ten most-highly differential gene targets in the lung of E17.5 $\mathrm{Creb} 1^{-1-}$ mice are shown. Genes with lower mRNA expression in $\mathrm{Creb} 1^{-1-}$ lungs relative to wildtype (putative Creb1-induced) are listed first in descending order of fold change, while genes with higher mRNA expression in Creb1 ${ }^{-1-}$ lungs relative to wildtype (putative Creb1-repressed) are listed beneath in ascending order of fold change.

doi:10.1371/journal.pone.0017843.t001

unclear. As phosphorylation of Crebl was detected primarily in distal epithelium at E18.5, and not in surrounding mesenchyme until E18.5, it is likely that Crebl mediates gene expression in a cell-autonomous manner to induce differentiation or specify the cell fate of distal epithelial populations, such as AECs, rather than via signalling from the adjacent mesenchyme. Indeed, the lack of Crebl expression we observed in the mesenchyme surrounding the epithelium at E16.5 may be a means to prevent activation of
A

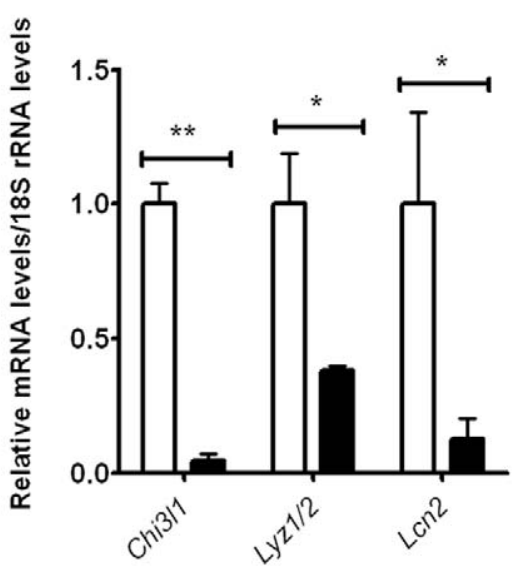

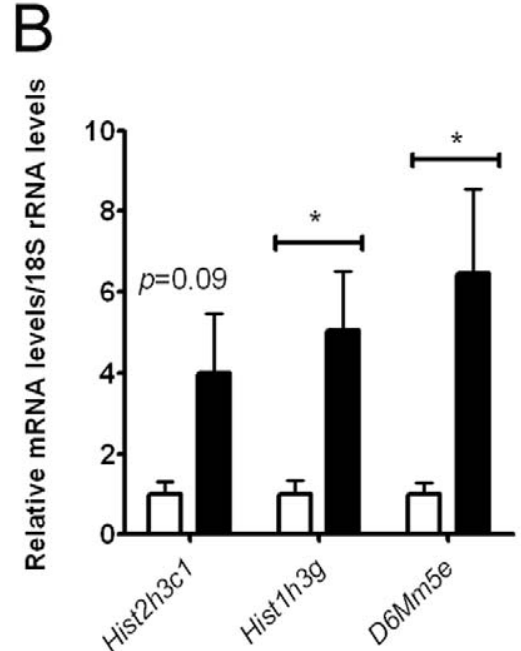

Figure 9. Gene expression analysis of highly-differential Creb1 microarray lung gene targets. qPCR analysis of mRNA levels for downregulated microarray gene targets: Chi3/1, Lyz1/2, and $L \mathrm{cn} 2$ in the lung of E17.5 Creb $1^{-1-}$ mice relative to wildtype $(n=4)(A)$. qPCR analysis of mRNA levels for up-regulated microarray gene targets: Hist2h3c1, Hist1h3g, and D6Mm5e in the lung of E17.5 Creb1 ${ }^{-1-}$ mice relative to wildtype ( $\left.n=4\right)(\mathrm{B})$. Error bars represent SEM. Asterisk $\left(^{*}\right)$ indicates $p<0.05$ and ${ }^{* *}$ indicates $p<0.001$. White bars: Wildtype, Black bars: Creb $1^{-1-}$. doi:10.1371/journal.pone.0017843.g009 
Creb1. However it is also likely that Creb1-mediated signalling in the distal epithelium is not required or much utilized after E16.5 as at E18.5, pCrebl was localised primarily to the mesenchymal cells within saccular walls. Crebl signalling from the mesenchyme beyond E18.5 may therefore have an important role in postnatal lung development. Although we find differentiation of proximal epithelium is also affected in lungs of $\mathrm{Creb}^{-1-}$ mice, we detected only sparse activation of Crebl in these cells. We suggest that perhaps Crebl is activated in these cells much earlier for a specific interval to promote cell differentiation, and then activation is repressed in the same way as pCrebl is eventually lost in distal epithelium by E18.5. Transient activation of Crebl by phosphorylation has also been reported in mouse neuroblasts during neuronal differentiation [34].

The genes regulated by Crebl in the developing lung are almost completely unknown. Several studies have shown that cAMP stimulates gene expression of surfactant protein mRNAs [35] however Crebl can be activated by signalling pathways other than those which are stimulated by cAMP. Our microarray analysis and qPCR data at E17.5 indicate that with the exception of Sftpd, surfactant protein mRNAs are not greatly affected by a loss of Creb1. Sftpd, together with Sftpal and serum mannose-binding protein are members of the calcium-dependent collagenous lectin (collectin) subfamily of mammalian C-type, lectins, and is involved with the immune response to inhaled pathogens and microorganisms [36]. Sftpd mRNA is initially expressed at E16 in mice [37], and its expression is limited to type-II AECs and non-ciliated bronchiolar epithelial cells which are most likely Clara cells $[38,39]$. In mice, we show Sftpd protein is localised to the conducting airway epithelium at E17.5. It is therefore possible that the very large reduction in Sftpd mRNA levels and also lack of Sftpd protein immunostaining in the Creb1 $1^{-/-}$lung we observe at E17.5 may be due to the absence of Scgblal-positive Clara cells which we detect at this stage of development. Alternatively Crebl may have an active role in Sftpd gene transcription since we also show a loss of Sftpd expression in non-lung tissues such as the stratum spinosum epidermal layer in E17.5 $\mathrm{Creb}^{-/-}$mice compared with robust expression in wildtype mice. Transcriptional regulation of the Sftpd promoter has not been thoroughly explored, however studies to date in human and mouse have revealed a role for AP-1 proteins [40], CCAAT enhancer-binding proteins (C/EBPs) [41] and Nuclear factor of activated $\mathrm{T}$ cells (NFATs) [42] in directing Sftpd gene expression.

As well as $S f t p d$, the microarray analysis detected a high number of genes involved with the immune response such as Chi3l1, Lyz1\&2, Lcn2, Scgb1a1, Hc, Vnn1, H2-10, Ier3, Tnfaip, Hp and C3 which are also highly under-expressed in the E17.5 Creb1 $^{-/-}$lung. This suggests a role for Crebl in stimulating expression of genes required for the regulation of the immune response in the developing lung. For example, Chi3ll promotes $\mathrm{T}$ cell helper 2 (Th2) -induced inflammation in response to an allergen challenge [43], Hc and C3 together constitute crucial elements of the complement system which mediates several immune responses to target foreign pathogens [44], while Lcn2 [45] and Lyz factors [46] have important roles in the immune response to bacterial infection. How Crebl-mediated stimulation of immune responseassociated genes is related to development of the lung is unclear, however interestingly the induction of pro-inflammatory mediators

\section{References}

1. Okubo T, Knoepfler PS, Eisenman RN, Hogan BL (2005) Nmyc plays an essential role during lung development as a dosage-sensitive regulator of progenitor cell proliferation and differentiation. Development 132: 1363-1374. has been correlated with increased maturation of the fetal lung close to term, and may even be a more potent accelerator of lung development than corticosteroid exposure [47]. Furthermore, very similar trends in expression of immune response-related genes has also been shown in the lungs of mice with targeted mutations of Nkx2-1 [48], Foxa2 [49], Clebp $\alpha$ [50] and Cnb1 [51], all of which exhibit a severe disruption in lung development at late gestation, similar to $C r e b 1^{-1-}$ mice. Microarray analyses of fetal lungs performed in these studies show that expression of genes involved in the immune response and host defence are among the most highly differential, usually with reduced expression compared to wildtype. It is even likely that $L y z 1$ and $L y z 2$ are direct transcriptional targets of Foxa2 [49]. Further studies are therefore needed to investigate the clear link between transcription factor signalling and genes involved with immune response in the context of lung development.

In conclusion, we show that Crebl-mediated signalling is required for proper differentiation of important epithelial cell populations in the developing lung including ciliated, Clara, and neuroendocrine cells in the proximal airway, as well as type-II and particularly type-I AECs of the distal epithelium. Further investigation will be needed to determine how the timing of Crebl phosphorylation in lung cell populations affect epithelial differentiation, both before and after birth, and also which genes are regulated by Crebl in the developing lung to promote its effects. It will be particularly interesting to determine the effects of a cell-type specific targeted deletion of Crebl in the respiratory epithelium. Additionally, it will be useful to identify the upstream signalling pathways responsible for phosphorylation of Crebl specifically in the developing lung, and also which circulating ligands stimulate these pathways.

\section{Supporting Information}

Figure S1 Greb1 protein is not detected in the lung of Creb1 $^{-1-}$ mice. Immunohistochemistry for Crebl protein in the lung of E18.5 wildtype and $C r e b 1^{-/-}$mice. Crebl is strongly detected in the lung of wildtype mice (A), but is almost completely absent in the lung of $\mathrm{Creb1}^{-/-}$mice (B). Scale bars: $100 \mu \mathrm{m}$. (TIF)

Table S1 Analysis of $\mathrm{Creb1}^{-/-}$mouse mortality in utero.

(DOC)

\section{Acknowledgments}

We thank Ian Boundy and XiaoWen (Partek) for expert technical assistance.

\section{Author Contributions}

Conceived and designed the experiments: ADB SBH TJC. Performed the experiments: ADB SJF KHT NA. Analyzed the data: ADB SJF KHT. Contributed reagents/materials/analysis tools: TM SBH TJC. Wrote the paper: ADB SJF SBH TJC. Revision of manuscript for important intellectual content and interpretation of data: PFO.

2. Perl AK, Wert SE, Nagy A, Lobe CG, Whitsett JA (2002) Early restriction of peripheral and proximal cell lineages during formation of the lung. Proc Natl Acad Sci U S A 99: 10482-10487. 
3. Maeda Y, Dave V, Whitsett JA (2007) Transcriptional control of lung morphogenesis. Physiol Rev 87: 219-244.

4. Hai T, Hartman MG (2001) The molecular biology and nomenclature of the activating transcription factor/cAMP responsive element binding family of transcription factors: activating transcription factor proteins and homeostasis. Gene 273: 1-11.

5. Daniel PB, Walker WH, Habener JF (1998) Cyclic AMP signaling and gene regulation. Annu Rev Nutr 18: 353-383.

6. Gonzalez GA, Montminy MR (1989) Cyclic AMP stimulates somatostatin gene transcription by phosphorylation of CREB at serine 133. Cell 59: 675-680.

7. Johannessen M, Delghandi MP, Moens U (2004) What turns CREB on? Cell Signal 16: 1211-1227.

8. Blendy JA, Kaestner KH, Weinbauer GF, Nieschlag E, Schutz G (1996) Severe impairment of spermatogenesis in mice lacking the CREM gene. Nature 380: $162-165$.

9. Bleckmann SC, Blendy JA, Rudolph D, Monaghan AP, Schmid W, et al. (2002) Activating transcription factor 1 and CREB are important for cell survival during early mouse development. Mol Cell Biol 22: 1919-1925.

10. Hummler E, Cole TJ, Blendy JA, Ganss R, Aguzzi A, et al. (1994) Targeted mutation of the CREB gene: compensation within the CREB/ATF family of transcription factors. Proc Natl Acad Sci U S A 91: 5647-5651.

11. Rudolph D, Tafuri A, Gass P, Hammerling GJ, Arnold B, et al. (1998) Impaired fetal $\mathrm{T}$ cell development and perinatal lethality in mice lacking the cAMP response element binding protein. Proc Natl Acad Sci U S A 95: 4481-4486.

12. Mantamadiotis T, Lemberger T, Bleckmann SC, Kern H, Kretz O, et al. (2002) Disruption of CREB function in brain leads to neurodegeneration. Nat Genet 31: $47-54$.

13. Pfaffl MW (2001) A new mathematical model for relative quantification in realtime RT-PCR. Nucleic Acids Res 29: e45.

14. Flecknoe S, Harding R, Maritz G, Hooper SB (2000) Increased lung expansion alters the proportions of type I and type II alveolar epithelial cells in fetal sheep. Am J Physiol Lung Cell Mol Physiol 278: L1180-1185.

15. Flecknoe SJ, Wallace MJ, Harding R, Hooper SB (2002) Determination of alveolar epithelial cell phenotypes in fetal sheep: evidence for the involvement of basal lung expansion. J Physiol 542: 245-253.

16. Flecknoe SJ, Boland RE, Wallace MJ, Harding R, Hooper SB (2004) Regulation of alveolar epithelial cell phenotypes in fetal sheep: roles of cortisol and lung expansion. Am J Physiol Lung Cell Mol Physiol 287: L1207-1214.

17. Flecknoe SJ, Wallace MJ, Cock ML, Harding R, Hooper SB (2003) Changes in alveolar epithelial cell proportions during fetal and postnatal development in sheep. Am J Physiol Lung Cell Mol Physiol 285: L664-670.

18. Sozo F, Wallace MJ, Hanna MR, Flecknoe SJ, Cock ML, et al. (2006) Alveolar epithelial cell differentiation and surfactant protein expression after mild preterm birth in sheep. Pediatr Res 59: 151-156.

19. Irizarry RA, Bolstad BM, Collin F, Cope LM, Hobbs B, et al. (2003) Summaries of Affymetrix GeneChip probe level data. Nucleic Acids Res 31: e15.

20. Irizarry RA, Hobbs B, Collin F, Beazer-Barclay YD, Antonellis KJ, et al. (2003) Exploration, normalization, and summaries of high density oligonucleotide array probe level data. Biostatistics 4: 249-264.

21. Bolstad BM, Irizarry RA, Astrand M, Speed TP (2003) A comparison of normalization methods for high density oligonucleotide array data based on variance and bias. Bioinformatics 19: 185-193.

22. Kerr MK, Martin M, Churchill GA (2000) Analysis of variance for gene expression microarray data. J Comput Biol 7: 819-837.

23. Storey JD, Tibshirani R (2003) Statistical significance for genomewide studies. Proc Natl Acad Sci U S A 100: 9440-9445.

24. Long F, Schipani E, Asahara H, Kronenberg H, Montminy M (2001) The CREB family of activators is required for endochondral bone development. Development 128: 541-550.

25. Fitzgerald ML, Xavier R, Haley KJ, Welti R, Goss JL, et al. (2006) ABCA3 inactivation in mice causes respiratory failure, loss of pulmonary surfactant and depletion of lung phosphatidylglycerol. J Lipid Res.

26. Liu Y, Hogan BL (2002) Differential gene expression in the distal tip endoderm of the embryonic mouse lung. Gene Expr Patterns 2: 229-233.

27. Zhou L, Lim L, Costa RH, Whitsett JA (1996) Thyroid transcription factor-1, hepatocyte nuclear factor-3beta, surfactant protein B, C, and Clara cell secretory protein in developing mouse lung. J Histochem Cytochem 44: 1183-1193.
28. Tichelaar JW, Wert SE, Costa RH, Kimura S, Whitsett JA (1999) HNF-3/ forkhead homologue-4 (HFH-4) is expressed in ciliated epithelial cells in the developing mouse lung. J Histochem Cytochem 47: 823-832.

29. Rawlins EL, Ostrowski LE, Randell SH, Hogan BL (2007) Lung development and repair: contribution of the ciliated lineage. Proc Natl Acad Sci U S A 104: $410-417$.

30. Matus M, Lewin G, Stumpel F, Buchwalow IB, Schneider MD, et al. (2007) Cardiomyocyte-specific inactivation of transcription factor CREB in mice. Faseb J 21: 1884-1892.

31. D'Amore-Bruno MA, Wikenheiser KA, Carter JE, Clark JC, Whitsett JA (1992) Sequence, ontogeny, and cellular localization of murine surfactant protein B mRNA. Am J Physiol 262: L40-47.

32. Evans MJ, Cabral LJ, Stephens RJ, Freeman G (1975) Transformation of alveolar type 2 cells to type 1 cells following exposure to NO2. Exp Mol Pathol 22: $142-150$.

33. Alcorn DG, Adamson TM, Maloney JE, Robinson PM (1981) A morphologic and morphometric analysis of fetal lung development in the sheep. Anat Rec 201: 655-667.

34. Giachino C, De Marchis S, Giampietro C, Parlato R, Perroteau I, et al. (2005) cAMP response element-binding protein regulates differentiation and survival of newborn neurons in the olfactory bulb. J Neurosci 25: 10105-10118.

35. Mendelson CR (2000) Role of transcription factors in fetal lung development and surfactant protein gene expression. Annu Rev Physiol 62: 875-915.

36. Forbes LR, Haczku A SP-D and regulation of the pulmonary innate immune system in allergic airway changes. Clin Exp Allergy 40: 547-562.

37. Wong CJ, Akiyama J, Allen L, Hawgood S (1996) Localization and developmental expression of surfactant proteins D and A in the respiratory tract of the mouse. Pediatr Res 39: 930-937.

38. Madsen J, Kliem A, Tornoe I, Skjodt K, Koch C, et al. (2000) Localization of lung surfactant protein D on mucosal surfaces in human tissues. J Immunol 164: 5866-5870

39. Akiyama J, Hoffman A, Brown C, Allen L, Edmondson J, et al. (2002) Tissue distribution of surfactant proteins $\mathrm{A}$ and $\mathrm{D}$ in the mouse. J Histochem Cytochem 50: 993-996.

40. He Y, Crouch EC, Rust K, Spaite E, Brody SL (2000) Proximal promoter of the surfactant protein D gene: regulatory roles of AP-1, forkhead box, and GT box binding proteins. J Biol Chem 275: 31051-31060.

41. He Y, Crouch E (2002) Surfactant protein D gene regulation. Interactions among the conserved CCAAT/enhancer-binding protein elements. J Biol Chem 277: 19530-19537.

42. Dave V, Childs T, Whitsett JA (2004) Nuclear factor of activated T cells regulates transcription of the surfactant protein D gene (Sftpd) via direct interaction with thyroid transcription factor-1 in lung epithelial cells. J Biol Chem 279: 34578-34588.

43. Lee CG, Hartl D, Lee GR, Koller B, Matsuura H, et al. (2009) Role of breast regression protein 39 (BRP-39)/chitinase 3-like-1 in Th2 and IL-13-induced tissue responses and apoptosis. J Exp Med 206: 1149-1166.

44. Guo RF, Ward PA (2005) Role of C5a in inflammatory responses. Annu Rev Immunol 23: 821-852.

45. Flo TH, Smith KD, Sato S, Rodriguez DJ, Holmes MA, et al. (2004) Lipocalin 2 mediates an innate immune response to bacterial infection by sequestrating iron. Nature 432: 917-921.

46. Markart P, Korfhagen TR, Weaver TE, Akinbi HT (2004) Mouse lysozyme M is important in pulmonary host defense against Klebsiella pneumoniae infection. Am J Respir Crit Care Med 169: 454-458.

47. Kramer BW, Kallapur S, Newnham J, Jobe AH (2009) Prenatal inflammation and lung development. Semin Fetal Neonatal Med 14: 2-7.

48. DeFelice M, Silberschmidt D, DiLauro R, Xu Y, Wert SE, et al. (2003) TTF-1 phosphorylation is required for peripheral lung morphogenesis, perinatal survival, and tissue-specific gene expression. J Biol Chem 278: 35574-35583.

49. Wan H, Xu Y, Ikegami M, Stahlman MT, Kaestner KH, et al, (2004) Foxa2 is required for transition to air breathing at birth. Proc Natl Acad Sci U S A 101: $14449-14454$

50. Martis PG, Whitsett JA, Xu Y, Perl AK, Wan H, et al. (2006) C/EBPalpha is required for lung maturation at birth. Development 133: 1155-1164.

51. Dave V, Childs T, Xu Y, Ikegami M, Besnard V, et al. (2006) Calcineurin/Nfat signaling is required for perinatal lung maturation and function. J Clin Invest 116: 2597-2609. 